\title{
Luminescent/magnetic PLGA-based hybrid nanocomposites: a smart nanocarrier system for targeted codelivery and dual-modality imaging in cancer theranostics
}

\author{
This article was published in the following Dove Press journal: \\ International Journal of Nanomedicine \\ 6 June 2017 \\ Number of times this article has been viewed
}

\author{
Xue Shen' \\ Tingting $\mathrm{Li}^{\prime}$ \\ Zhongyuan Chen' \\ Yue Geng' \\ Xiaoxue Xie' \\ Shun $\mathrm{Li}^{1,2}$ \\ Hong Yang ${ }^{1,2}$ \\ Chunhui $\mathrm{Wu}^{1,2}$ \\ Yiyao Liu ${ }^{1,2}$ \\ 'Department of Biophysics, School of \\ Life Science and Technology, ${ }^{2}$ Center \\ for Information in Biology, University \\ of Electronic Science and Technology \\ of China, Chengdu, Sichuan, \\ People's Republic of China
}

\begin{abstract}
Cancer diagnosis and treatment represent an urgent medical need given the rising cancer incidence over the past few decades. Cancer theranostics, namely, the combination of diagnostics and therapeutics within a single agent, are being developed using various anticancer drug-, siRNA-, or inorganic materials-loaded nanocarriers. Herein, we demonstrate a strategy of encapsulating quantum dots, superparamagnetic $\mathrm{Fe}_{3} \mathrm{O}_{4}$ nanocrystals, and doxorubicin (DOX) into biodegradable poly(D,L-lactic-co-glycolic acid) (PLGA) polymeric nanocomposites using the double emulsion solvent evaporation method, followed by coupling to the amine group of polyethyleneimine premodified with polyethylene glycol-folic acid (PEI-PEG-FA [PPF]) segments and adsorption of vascular endothelial growth factor (VEGF)-targeted small hairpin RNA (shRNA). VEGF is important for tumor growth, progression, and metastasis. These drug-loaded luminescent/magnetic PLGA-based hybrid nanocomposites (LDM-PLGA/PPF/VEGF shRNA) were fabricated for tumor-specific targeting, drug/gene delivery, and cancer imaging. The data showed that LDM-PLGA/PPF/VEGF shRNA nanocomposites can codeliver DOX and VEGF shRNA into tumor cells and effectively suppress VEGF expression, exhibiting remarkable synergistic antitumor effects both in vitro and in vivo. The cell viability was $\sim 14 \%$ when treated with LDM-PLGA/PPF/VEGF shRNA nanocomposites ([DOX] $=25 \mu \mathrm{g} / \mathrm{mL}$ ), and in vivo tumor growth data showed that the tumor volume decreased by $81 \%$ compared with the saline group at 21 days postinjection. Magnetic resonance and fluorescence imaging data revealed that the luminescent/magnetic hybrid nanocomposites may also be used as an efficient nanoprobe for enhanced $T_{2}$-weighted magnetic resonance and fluorescence imaging in vitro and in vivo. The present work validates the great potential of the developed multifunctional LDM-PLGA/PPF/ VEGF shRNA nanocomposites as effective theranostic agents through the codelivery of drugs/ genes and dual-modality imaging in cancer treatment.
\end{abstract}

Keywords: doxorubicin, codelivery, dual-modality imaging, synergistic antitumor effects, VEGF shRNA

\section{Introduction}

Cancer represents a major public health problem worldwide, gradually becoming the leading cause of morbidity and mortality. ${ }^{1-3}$ Chemotherapeutic treatment is the most common type of cancer therapy. ${ }^{4}$ Doxorubicin (DOX), a widely used anticancer drug approved by the Food and Drug Administration (FDA), is a first-line chemotherapeutic agent used, alone or in combination with other agents, in the treatment of various solid tumors, including breast, ovary, bladder, cervix, prostate, and lung cancers, and
Department of Biophysics, School of Life Science and Technology, University of Electronic Science and Technology of China, Chengdu 610054, Sichuan, People's Republic of China

Tel +86 2883203353

Fax +86288320 8238

Email liuyiyao@uestc.edu.cn;

liuyiyao@hotmail.com 
lymphoblastic leukemia. ${ }^{5}$ However, the clinical applications of DOX remain limited due to its severely toxic side effects, including cardiotoxicity, myelosuppression, mucositis, and alopecia, caused by its low specificity to cancer cells and dose-dependent toxicity to healthy organs. ${ }^{6,7}$ In addition, many tumor cells can develop drug resistance, resulting in lower drug concentrations in tumor cells and weakening/ diminishing their therapeutic efficacy. ${ }^{8}$ Thus, there is an urgent need to develop treatment strategies able to maximize the therapeutic efficacy and minimize the adverse effects of the current chemotherapeutics. ${ }^{9,10}$

Magnetic resonance (MR) imaging has been widely used as a powerful imaging technique in clinical applications due to its high-resolution and tomographic capabilities. ${ }^{11,12}$ Positive and negative contrast agents are the two major types of MR contrast agents; examples of these include gadoliniumbased $T_{1}$ contrast agents ${ }^{13,14}$ and superparamagnetic $\mathrm{Fe}_{3} \mathrm{O}_{4}$ nanocrystal-based $T_{2}$ contrast agents. ${ }^{12,15,16}$ Superparamagnetic $\mathrm{Fe}_{3} \mathrm{O}_{4}$ nanoparticles have been approved for clinical diagnosis by the $\mathrm{FDA}^{17}$ and have been the focus of various studies as negative contrast agents given their high magnetic responsiveness. ${ }^{18,19}$ Another imaging modality is quantum dot (QD)-based fluorescence imaging, which has been extensively studied in multicolor imaging of cells, tissues, and animals. ${ }^{20}$ As an emerging fluorescent contrast agent, QDs exhibit remarkable fluorescence intensity, a broad excitation wavelength range, and a narrow emission spectrum, all of which can be exploited in biomedical applications such as the detection of disease sites and reporting of therapy response. ${ }^{21,22}$ Thus, the combined use of magnetic nanoparticles with fluorescent dyes, especially QDs, could function as dual-modality imaging schemes for enhanced $T_{2}$-weighted MR imaging and tumor fluorescence imaging in vitro and in vivo. ${ }^{23}$ Nevertheless, $\mathrm{Fe}_{3} \mathrm{O}_{4}$ nanoparticles and QDs must be precoated with proper matrix to endow them with the properties of colloidal stability, biocompatibility, long blood circulation times, and nontoxicity in the physiological environment. ${ }^{24}$ On account of these challenges, numerous recent studies have focused on the manufacturing of various multifunctional nanocarrier systems combined with other inorganic nanocrystals, such as QDs, gold nanocrystals, magnetic particles, and carbon nanotubes, ${ }^{25-28}$ and an appropriate matrix, mainly based on polymers, lipids, and inorganic and natural materials. ${ }^{29-32}$ These systems have also been used as drug delivery systems for therapeutic purposes. ${ }^{33}$

The technique of RNA interference (RNAi), based on small interfering RNA (siRNA), has been extensively used as a tumor-specific gene therapy able to effectively downregulate the expression of targeted genes and is considered the most promising method for cancer treatment when combined with chemotherapy. ${ }^{34}$ However, naked siRNA molecules are vulnerable to degradation by nucleases in plasma before being able to reach the tumor tissue. ${ }^{35}$ Furthermore, it is extremely difficult for naked siRNA molecules to penetrate across the cell membranes given their hydrophilicity, high molecular weight, and high charge density. ${ }^{36}$ Therefore, the development of safe and effective siRNA delivery systems is essential for RNAi therapeutics. A feasible target for RNAi therapeutics is vascular endothelial growth factor (VEGF), a vital regulatory cytokine secreted by cancer cells during angiogenesis ${ }^{37,38}$ and important for tumor survival, growth, migration, and metastasis. ${ }^{39-41}$ RNAi-mediated silencing of VEGF expression has been validated as a feasible and effective cancer treatment, ${ }^{42-44}$ especially when combined with chemotherapeutic drugs within the same delivery system. ${ }^{45}$ Feng et al ${ }^{46}$ designed a core-shell type nanoparticle system embedded by vapreotide-modified polyethylene glycol phospholipid vapreotide to deliver VEGF siRNA and paclitaxel, resulting in enhanced tumor therapy efficacy through tumor-targeted delivery, cytotoxicity of paclitaxel, and VEGF downregulation by siRNA silencing.

Given the previously mentioned challenges, herein, we designed a multifunctional poly(D,L-lactic-co-glycolic acid) (PLGA)-based luminescent/magnetic hybrid nanocomposite modified with polyethyleneimine premodified with polyethylene glycol-folic acid (PEI-PEG-FA, [PPF]) segments to codeliver DOX and VEGF small hairpin RNA (shRNA) (LDM-PLGA/PPF/VEGF shRNA). The PEGconjugated copolymer (PPF) was used to prevent particles from aggregation and to achieve a long circulation time in vivo. ${ }^{47}$ PLGA was chosen due to its biocompatibility and biodegradability. ${ }^{48}$ The LDM-PLGA/PPF/VEGF shRNA nanocomposites had a dual targeting function through folate receptor-mediated targeting and magnetic targeting. As illustrated in Figure 1, the PPF copolymer was initially synthesized, followed by further modification on the surface of LDM-PLGA nanoparticles prepared by a double emulsion solvent evaporation method, and finally by electrostatic adsorption of VEGF shRNA, forming the theranostic agent (LDM-PLGA/PPF/VEGF shRNA). The drug release behavior, folate receptor-mediated cell uptake, cytotoxicity, escape from endosomes/lysosomes, gene expression, MR and fluorescence imaging, and antitumor effects in an animal model of the developed nanocomposites were investigated. It was expected that the as-synthesized PLGA-based multifunctional nanocomposites may provide a promising potential to act as an efficient codelivery system for theranostic applications. 


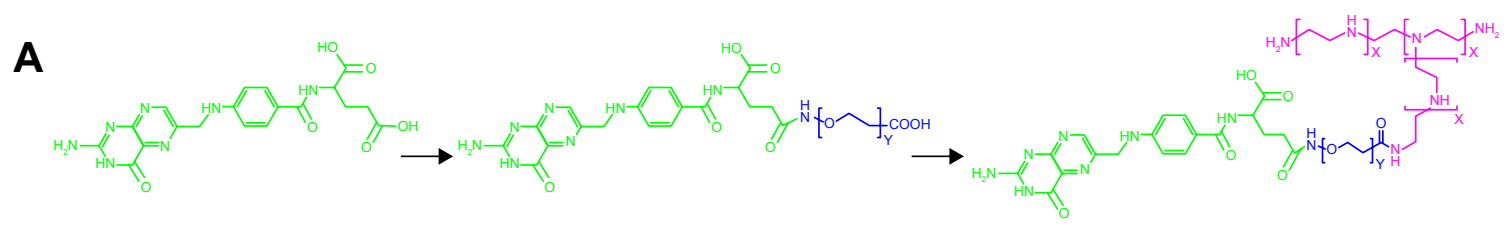

FA

FA-PEG-COOH

PEI-PEG-FA

B

PLGA
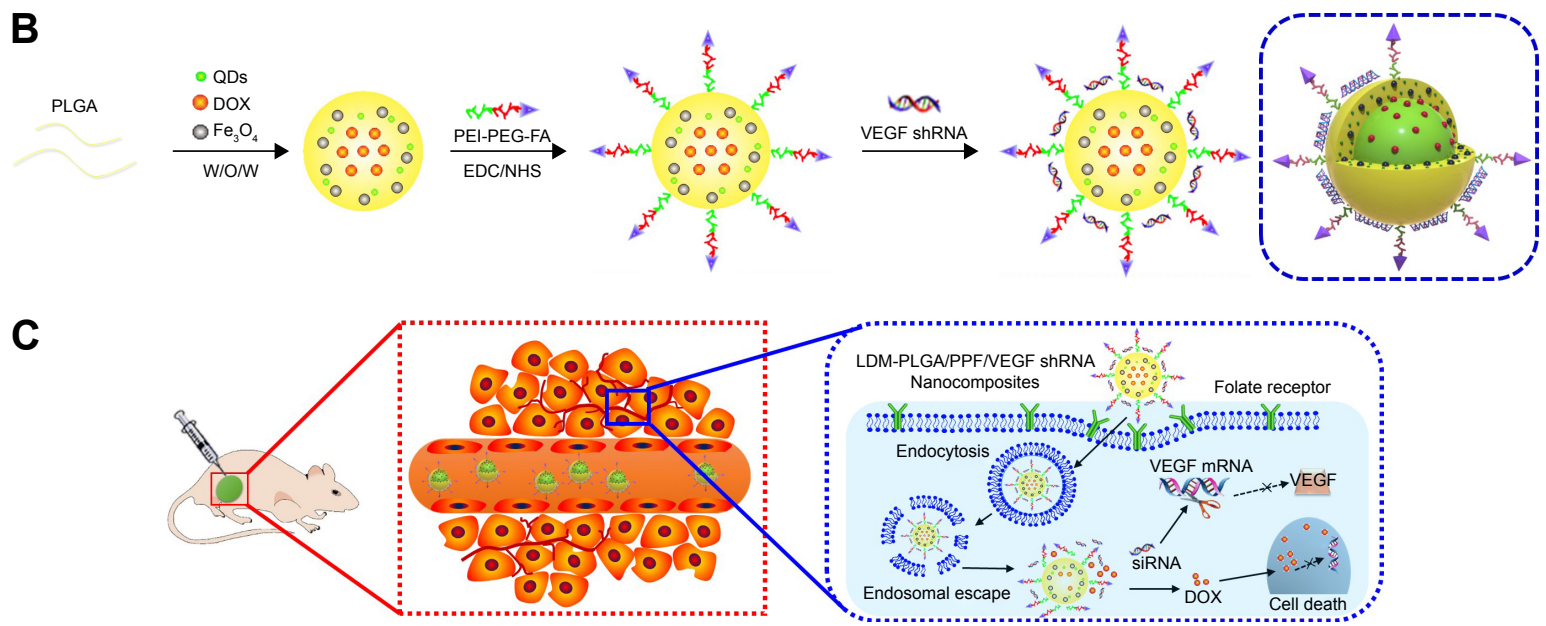

Figure I Schematic illustration of the synthesis procedure of LDM-PLGA/PPF/VEGF shRNA nanocomposites for codelivery of DOX and VEGF shRNA in EMT-6 tumor models.

Notes: (A) The preparation process of PEI-PEG-FA. (B) The construction of PLGA-based polymeric nanoparticles using a double emulsion solvent evaporation method. (C) The transport process of LDM-PLGA/PPF/VEGF shRNA nanocomposites and inhibition of tumor growth through cellular uptake via endocytosis, endosomal escape, intracellular VEGF shRNA, and DOX release.

Abbreviations: DOX, doxorubicin; PLGA, poly(D,L-lactic-co-glycolic acid); PEI-PEG-FA, polyethyleneimine premodified with polyethylene glycol-folic acid; PPF, PEIPEG-FA; shRNA, small hairpin RNA; VEGF, vascular endothelial growth factor; siRNA, small interfering RNA; QDs, quantum dots; EDC, N-(3-dimethylaminopropyl)-N'ethylcarbodiimide hydrochloride; NHS, N-hydroxysuccinimide.

\section{Materials and methods Materials}

PLGA (50:50, inherent viscosity $0.20 \mathrm{dL} / \mathrm{g}, \mathrm{MW}=15,000$ ) was obtained from Shandong Institute of Medical Instruments (Shandong, People's Republic of China), poly(vinyl alcohol) (PVA) (MW =30,000-70,000, 78\%-90\% hydrolyzed), branched polyethylenimine (PEI, $25 \mathrm{kDa}), \mathrm{N}-(3-$ dimethylaminopropyl)- $N^{\prime}$-ethylcarbodiimide hydrochloride (EDC), and $N$-hydroxysuccinimide (NHS) were purchased from Sigma-Aldrich (St Louis, MO, USA). PEG with one end of amine group and the other end of carboxyl group $\left(\mathrm{NH}_{2}-\mathrm{PEG}-\right.$ $\mathrm{COOH}, \mathrm{MW}=3,500$ ) was purchased from Shanghai Seebio Biotech, Inc. (Shanghai, People's Republic of China). $\mathrm{Fe}_{3} \mathrm{O}_{4}$ nanoparticles $(10 \mathrm{~nm})$ were obtained from Nanjing Emperor Nano Material Co. Ltd. (Nanjing, People's Republic of China), and CdSe/ZnS QDs were purchased from Wuhan Jiayuan Quanum Dots Co. Ltd. (Wuhan, People's Republic of China). Folic acid (FA) was obtained from Alexis (Los Angeles, CA, USA), and doxorubicin hydrochloride (DOX) was from Hisun Pharmaceutical (Zhejiang, People's Republic of China). CellTiter $96^{\circledR}$ AQueous One Solution Cell Proliferation Assay was purchased from Promega (Fitchburg, WI, USA). Calcein AM was purchased from Life Technologies (Grand Island, NY, USA). Cell culture medium Roswell Park Memorial Institute 1640, fetal bovine serum and trypsin were obtained from Gibco (Grand Island, NY, USA). All other chemicals and solvents, if not mentioned, were of analytical grade and used as received without additional purification. Plasmid-expressing shRNA against VEGF (VEGF shRNA) and scrambled shRNA (SC shRNA) were obtained from Fungenome Co. Ltd. (Guangzhou, People's Republic of China). The shRNA-targeted VEGF sequence is "TACTGCCATCCAATCGAGA". And the human umbilical vein endothelial cells (HUVECs), human cervical carcinoma cells (HeLa), and murine mammary cancer cells (EMT-6) were obtained from the American Type Culture Collection (Manassas, VA, USA).

\section{Synthesis of PPF}

Copolymer of PPF was synthesized as follows: FA (20 mg, $0.045 \mathrm{mM})$ was activated in the presence of EDC $(7.4 \mathrm{mg}$, $0.038 \mathrm{mM})$ and NHS (5.8 $\mathrm{mg}, 0.05 \mathrm{mM}$ ) by gentle stirring (300 rpm) in dimethyl sulfoxide (DMSO, $5 \mathrm{~mL}$ ) for $12 \mathrm{~h}$ at room temperature $\left(25^{\circ} \mathrm{C}\right)$; the resulting solution of activated FA was then dropwise added into the DMSO solution $(5 \mathrm{~mL})$ of $\mathrm{NH}_{2}$-PEG-COOH (MW =3,400, $36 \mathrm{mg}, 0.01 \mathrm{mM}$ ) under 
another $72 \mathrm{~h}$ gentle stirring. The reaction mixture was then dialyzed for $72 \mathrm{~h}$ against deionized water to remove any unreacted reactants using a dialysis membrane with a molecular weight cutoff of 1,000 Da. The resulting copolymer was freeze-dried to obtain the product FA-PEG-COOH. Then, the obtained FA-PEG-COOH was activated using EDC/ NHS in DMSO $(5 \mathrm{~mL})$ by gentle stirring for $3 \mathrm{~h}$, followed by coupling with PEI (32 mg, $0.001 \mathrm{mM}$ ) to allow for another $72 \mathrm{~h}$ gentle stirring. The reaction mixture was then dialyzed against deionized water using a dialysis membrane with a molecular weight cutoff of 8,000-14,000 Da for $72 \mathrm{~h}$ to remove the excess reactants, followed by freeze-drying to obtain the copolymer PPF.

\section{Fabrication of LDM-PLGA/PPF/VEGF shRNA nanocomposites}

LDM-PLGA nanoparticles were prepared by the water-in-oilin-water double emulsion method according to our previously published procedures with some modifications. ${ }^{49,50}$ Briefly, PLGA (100 mg) was directly dissolved in $2 \mathrm{~mL}$ methylene chloride with quantitative $\mathrm{Fe}_{3} \mathrm{O}_{4}$ nanoparticles and $\mathrm{CdSe} / \mathrm{ZnS}$ QDs dispersed homogeneously in the mixed solution, then $0.2 \mathrm{~mL}$ of DOX aqueous solution ( $6 \mathrm{mg}$ ) was added to the organic phase; subsequently, the mixture was emulsified by sonication with $45 \%$ amplitude for 2 min using Digital Sonifier S-250D (Branson Ultrasonic, Danbury, CT, USA) in an ice bath to obtain the primary emulsion (first emulsion). The primary emulsion was immediately poured into $10 \mathrm{~mL}$ of a PVA solution $(1 \%, \mathrm{w} / \mathrm{v})$ and sonicated for $1 \mathrm{~min}$ to form a double emulsion (second emulsion). The final emulsion was stirred at room temperature overnight to evaporate the organic solvent. The obtained LDM-PLGA nanoparticles were further collected by centrifugation at $12,000 \mathrm{rpm}$ for $10 \mathrm{~min}$, then washed three times with deionized water. Drug encapsulation efficiency (percentage of the actually encapsulated DOX out of that used to prepare LDM-PLGA nanoparticles) was measured using UV-visible spectroscopy (UV-2910; Hitachi, Tokyo, Japan) at $480 \mathrm{~nm}$. The supernatants of the emulsion suspension after centrifugation and after three washing steps were collected and quantified for the analysis of DOX encapsulation efficiency. The encapsulation efficiency of the drug was calculated by the following formula:

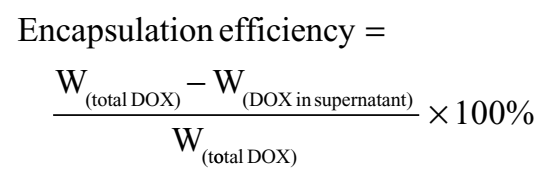

where $\mathrm{W}$ is the weight.
The obtained LDM-PLGA nanoparticles were further modified with the copolymer PPF. PPF was covalently coupled to the LDM-PLGA by EDC/NHS method. First, the carboxylic groups on the surfaces of LDM-PLGA were activated in the presence of EDC/NHS by gentle stirring (300 rpm) in aqueous solution for $1 \mathrm{~h}$. Then the PPF solution was added to the activated LDM-PLGA suspension (a weight ratio of 24:1 of LDM-PLGA to PPF), which was placed to adequately react in a temperature-controllable shaker $\left(120 \mathrm{rpm}, 37^{\circ} \mathrm{C}\right)$ for $4 \mathrm{~h}$ in a dark condition to allow PPF to graft onto the surface of the LDM-PLGA nanoparticles. Unbound PPF was removed by centrifugation at 12,000 rpm for $10 \mathrm{~min}$ followed by five washes with deionized water to obtain the resulting LDM-PLGA/PPF nanoparticles. VEGF shRNA was incubated in the LDM-PLGA/PPF suspension at various weight ratios $(100: 1,150: 1,200: 1$, and 300:1) for $30 \mathrm{~min}$ at room temperature to form the LDM-PLGA/PPF/ VEGF shRNA nanocomposites by electrostatic absorption. Then the LDM-PLGA/PPF/VEGF shRNA nanocomposites were loaded on a $1 \%$ agarose gel with tris/acetate/EDTA buffer and run at $120 \mathrm{~V}$ for $30 \mathrm{~min}$, followed by visualization by staining with ethidium bromide. Images were acquired using a UV transilluminator (Bio-Rad, Philadelphia, PA, USA). The non-DOX-loaded nanoparticles (LM-PLGA/ PPF or LM-PLGA/PPF/VEGF shRNA) were also prepared in the same manner.

\section{Characterization}

The size and morphology of the nanoparticles were examined by transmission electron microscopy (TEM) (JEM-100CX; JEOL, Tokyo, Japan) and scanning electron microscopy (SEM) (Helios NanoLab 650; FEI, Eindhoven, the Netherlands). The zeta-potentials and particle size distributions were determined using a ZetaPlus particle size and a zeta potential analyzer (Brookhaven Instruments, Holtsville, NY, USA) in water at $25^{\circ} \mathrm{C}$ in accordance with the manufacturer's operating manual. The ${ }^{1} \mathrm{H}$ NMR spectra of the PEG-FA and PPF were recorded using a Bruker AVANCE 600 nuclear magnetic resonance spectrometer (Bruker, Fällanden, Switzerland). The lyophilized PEG-FA and PPF were dissolved in deuterated DMSO (DMSO- $d_{6}$ ) and $\mathrm{D}_{2} \mathrm{O}$, respectively, before measurements.

\section{Hemolysis assay}

Red blood cells (RBCs) from healthy Sprague-Dawley rats were collected by centrifugation at $1,500 \mathrm{rpm}$ in heparincoated tubes. The upper plasma was removed, and the RBCs were then washed three times with sterile isotonic $0.9 \%$ 
$\mathrm{NaCl}$ solution. Following the last wash, the RBCs were resuspended with sterile isotonic $0.9 \% \mathrm{NaCl}$. The $\mathrm{RBC}$ suspension $(300 \mu \mathrm{L})$ was mixed with $900 \mu \mathrm{L}$ of 50,100 , 200,300 , and $400 \mu \mathrm{g} / \mathrm{mL}$ of LDM-PLGA and LDM-PLGA/ PPF suspension, respectively. The RBC suspensions $(300 \mu \mathrm{L})$ were added to $900 \mu \mathrm{L}$ deionized water as a positive control and $900 \mu \mathrm{L}$ sterile isotonic $0.9 \% \mathrm{NaCl}$ as a negative control. All samples were incubated at $37^{\circ} \mathrm{C}$ for $2 \mathrm{~h}$, and then centrifuged for $2 \mathrm{~min}$ at 4,000 rpm. The absorbance of the supernatant was measured at $541 \mathrm{~nm}$ using a UV-vis spectroscopy (UV-2910; Hitachi). The percentage hemolysis value was calculated using the following equation:

$$
\begin{aligned}
& \text { Hemolysis }(\%)= \\
& \frac{\text { Sample absorbance }- \text { Negative control }}{\text { Positive absorbance }- \text { Negative control }} \times 100 \%
\end{aligned}
$$

\section{Cell viability assay}

The cell viability of HeLa, EMT-6, and HUVEC after treatment with different nanoparticles was determined by CellTiter 96 AQueous One Solution Cell Proliferation Assay (MTS) (Promega) in accordance with manufacturer's instruction. Specifically, HeLa, EMT-6, and HUVEC cells were plated in a 96 -well plate at a density of $8 \times 10^{3}$ cells per well and incubated at $37^{\circ} \mathrm{C}$ in a $5 \% \mathrm{CO}_{2}$ atmosphere for $24 \mathrm{~h}$. After removing culture medium, HeLa and HUVEC cells were treated with $200 \mu \mathrm{L}$ of fresh medium containing LM-PLGA/PPF at a series of concentrations $(50,100,150$, 200 , and $250 \mu \mathrm{g} / \mathrm{mL}$ ) for 48 and $72 \mathrm{~h}$. Meanwhile, the other HeLa and EMT-6 cells were incubated with $200 \mu \mathrm{L}$ of fresh medium containing free DOX, LDM-PLGA/PPF (DOX concentration 10,15 , and $20 \mu \mathrm{g} / \mathrm{mL}$ ) and DOX-free PLGA nanoparticles (LM-PLGA/PPF) for $48 \mathrm{~h}$ with or without preincubating with free FA $(1.25 \mathrm{mM})$. Then the cells were washed at least three times using phosphate-buffered solution (PBS) and further incubated with $100 \mu \mathrm{L}$ fresh medium containing $10 \mu \mathrm{L}$ of MTS. The absorbance of each well was recorded at $490 \mathrm{~nm}$ using a microplate reader (ELx808; BioTek Instruments). All experiments were performed in triplicate. The relative cell viability was calculated by the following equation:

$$
\begin{aligned}
& \text { Relative cell viability }(\%)= \\
& \frac{\mathrm{A}_{490 \text { (treated) }}-\mathrm{A}_{490 \text { (blank) }}}{\mathrm{A}_{490 \text { (untreated) }}-\mathrm{A}_{490 \text { (blank) }}} \times 100 \%
\end{aligned}
$$

where $\mathrm{A}_{490}$ is the absorbance at $490 \mathrm{~nm}$ wavelength.

\section{Calcein-AM/PI costaining}

HeLa cells were seeded in a 24 -well plate at a density of $8 \times 10^{4}$ cells per well and incubated at $37^{\circ} \mathrm{C}$ in a $5 \% \mathrm{CO}_{2}$ atmosphere for $24 \mathrm{~h}$ to allow the cells to adhere and grown to $80 \%-90 \%$ confluence. After treatment with $20 \mu \mathrm{g} / \mathrm{mL}$ of DOX-loaded PLGA (LDM-PLGA/PPF) for $6 \mathrm{~h}$ with or without preincubating with free FA $(1.25 \mathrm{mM})$, the cells were rinsed three times with PBS, trypsinized, and centrifuged, and the defined amount of them was resuspended in $100 \mu \mathrm{L}$ PBS, in which live and dead cells were then costained by a mixture of calcein-AM $(1 \mathrm{mM})$ and PI $(1 \mathrm{mg} / \mathrm{mL})$ solution for $20 \mathrm{~min}$. Afterward, fluorescence microscopic images of cells were taken using an inverted fluorescent microscope (Nikon TE-2000U, Tokyo, Japan).

\section{Cellular experiments}

To observe the cellular uptake and intracellular drug release behaviors, HeLa cells were seeded into 24-well plates at a density of $8 \times 10^{4}$ cells per well and incubated for $24 \mathrm{~h}$ at $37^{\circ} \mathrm{C}$ in a $5 \% \mathrm{CO}_{2}$ atmosphere to allow cell attachment. The medium was then replaced with fresh medium containing DOX and DM-PLGA DM-PLGA/PPF (DOX concentration $5 \mu \mathrm{g} / \mathrm{mL}$ ) and incubated at $37^{\circ} \mathrm{C}$ for different times. Before imaging, the cells were rinsed three times with PBS (pH 7.4) and fixed with 4\% paraformaldehyde for $20 \mathrm{~min}$ and further labeled with 4,6-diamino-2-phenylindole (DAPI). The fluorescence of cells was visualized with a confocal laser scanning microscopy (CLSM; Leica SP5II, Wetzlar, Germany).

For magnetic targeting and folate targeting assay, HeLa cells were seeded into $35-\mathrm{mm}$ confocal dishes at a density of $15 \times 10^{4}$ cells per dish or a 24 -well plate at a density of $8 \times 10^{4}$ cells per well and incubated in complete medium for $24 \mathrm{~h}$ at $37^{\circ} \mathrm{C}$ in a $5 \% \mathrm{CO}_{2}$ atmosphere. Then the DM-PLGA/ PPF nanoparticles (DOX concentration $5 \mu \mathrm{g} / \mathrm{mL}$ ) were added to the dish or plate. A magnet was then placed at the bottom of the cell culture dish. For FA competition experiments, free FA (1.25 mM) was added to HeLa cells and preincubating for $2 \mathrm{~h}$ prior to the addition of nanoparticles. After incubation for $6 \mathrm{~h}$, the cells were washed with PBS ( $\mathrm{pH} 7.4)$ three times and fixed with 4\% paraformaldehyde for $20 \mathrm{~min}$ and further labeled with DAPI. Then images were examined under an inverted fluorescent microscope (Nikon TE-2000U). For quantitative analysis, cells were lysed with $0.5 \%(\mathrm{w} / \mathrm{v})$ sodium dodecyl sulfate ( $\mathrm{pH} 8.0$ ), and the DOX fluorescence intensity was subsequently detected using a fluorospectrophotometer (Hitachi F-7000). 
For nanoparticle intracellular distribution assay, HeLa cells were treated with fresh medium containing DMPLGA/PPF (DOX concentration $5 \mu \mathrm{g} / \mathrm{mL}$ ) and incubated at $37^{\circ} \mathrm{C}$ for 1,2 , or $4 \mathrm{~h}$, and further incubated with $100 \mathrm{nM}$ LysoTracker Green for 20 min or 200 nM MitoTracker Green for $30 \mathrm{~min}$ to stain lyosomes and mitochondria, respectively, and the excitation wavelengths of LysoTracker Green and MitoTracker Green were both $488 \mathrm{~nm}$. The images were acquired by CLSM.

\section{In vitro gene silencing effect}

HeLa cells were seeded in 12 -well culture plates at a density of $1.5 \times 10^{5}$ per well and cultured for $24 \mathrm{~h}$ to allow the cells to adhere and grown to $30 \%-40 \%$ confluence. And the cells were further treated with PLGA/PPF/VEGF shRNA, PLGA/ PPF/SC shRNA, and Lipo/VEGF shRNA (Lipofectamine 2000, a commercial transfection reagent) as a positive control. The dosage of VEGF shRNA was $2 \mu \mathrm{g}$, which was incubated with the nanoparticles at a weight ratio of 200:1 for $30 \mathrm{~min}$ before adding to the plates. The fresh cell culture medium was added after incubation in serum-free medium for $6 \mathrm{~h}$. The cell culture medium was collected after incubation for another $72 \mathrm{~h}$, followed by centrifugation at 7,000 rpm for $5 \mathrm{~min}$, in which the concentration of VEGF protein was analyzed by a human VEGF enzymelinked immunosorbent assay (ELISA) kit (NeoBioscience, Shenzhen, People's Republic of China). The VEGF mRNA level in the transfected HeLa cells was evaluated by quantitative real-time polymerase chain reaction (qRT-PCR) assay. One microgram aliquot of total mRNA was transcribed into complementary DNA using the PrimeScript RT Reagent Kit (Takara, Dalian, People's Republic of China). SYBR ${ }^{\circledR}$ Premix Ex Taq ${ }^{\mathrm{TM}}$ II was utilized to perform qRT-PCR on a real-time PCR system (CFX96 Real-Time PCR Detection System; Bio-Rad). And the amplification threshold $\left(\mathrm{C}_{t}\right)$ of each gene was normalized to that of glyceraldehyde 3-phosphate dehydrogenase (GAPDH). The comparative $\mathrm{C}_{t}$ method was used to calculate the fold changes. The efficiency of all primer pairs was $95 \%-100 \%$. Primer pairs used were VEGF (forward: 5'-TTCTCAAGGACCACCGCATC-3';

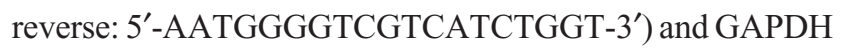
(forward: 5'-GTCTCCTCTGACTTCAACAGCG-3'; reverse: 5'-ACCACCC TGTTGCTGTAGCCAA-3').

\section{Animals and tumor model}

Female BALB/c mice, 5-6 weeks of age with the average weight of $20 \mathrm{~g}$, were provided by Laboratory Animal Centre of Sichuan University (Chengdu, People's Republic of China). Mice were maintained in a specific pathogen-free animal house under a 12:12 h light-dark cycle. All animal experiments were performed in agreement with the guidelines of the Institutional Animal Care Committee of the University of Electronic Science and Technology of China (UESTC). The Institutional Animal Care Committee of UESTC have approved the animal experimental procedures and protocols before experiments (approval number UESTC-AEC20160156, February $20,2016)$. The BALB/c mice were subcutaneously injected with $\sim 2 \times 10^{6}$ cells $/$ mouse at the right back region. When the tumor size grew to a volume of $80-100 \mathrm{~mm}^{3}$ at 5-6 days postinjection (volume $=$ length $\times$ width $^{2} / 2$ ), the mice were subsequently used for the following experiments.

\section{MR imaging in vitro and in vivo}

$T_{2}$-weighted MR signals of the LDM-PLGA/PPF in HeLa cells with various nanoparticle concentrations were measured. HeLa cells were seeded at a density of $4 \times 10^{5}$ per well on 12-well plates and cultured overnight. Then the culture medium was refreshed with the complete culture medium containing LDM-PLGA/PPF at a series of concentrations $(80,100,150,200$, and $300 \mu \mathrm{g} / \mathrm{mL})$ with Fe concentrations $(1.8,2.3,3.4,4.6$, and $6.8 \mu \mathrm{g} / \mathrm{mL})$ for $12 \mathrm{~h}$. The medium without any nanoparticles was used as the control. After that, the cells were rinsed three times with PBS, trypsinized, and centrifuged, and all of them were resuspended in $500 \mu \mathrm{L}$ of $1 \%$ agarose in 1.5 -mL Eppendorf centrifuge tubes. Moreover, MR imaging was acquired at $25^{\circ} \mathrm{C}$ by using a clinical 3.0 Tesla Clinical Siemens Trio scanner (Discovery MR750; GE Healthcare Bio-Sciences Corp., Piscataway, NJ, USA).

For in vivo MR imaging, the BALB/c mice bearing EMT-6 tumors were administered an intratumoral injection of LDM-PLGA/PPF (50 $\mu \mathrm{L}, 27.4 \mu \mathrm{g}$ Fe per mouse). Before MR imaging, the mice were anesthetized by intraperitoneal injection of sodium pentobarbital $(50 \mathrm{mg} / \mathrm{kg})$. Then MR imaging was carried out before and $12 \mathrm{~h}$ after injection by using a clinical 3.0 Tesla Clinical Siemens Trio scanner, and the sequence was repetition time $=8,000 \mathrm{~ms}$, echo time $=104.3 \mathrm{~ms}$, and slice thickness $=2 \mathrm{~mm}$.

\section{Fluorescence imaging studies}

For in vivo fluorescence imaging, the BALB/c nude mice bearing EMT-6 tumors were administered an intratumoral injection of L-PLGA/PPF $(50 \mu \mathrm{L}, 120 \mu \mathrm{g} / \mathrm{mL}$ of QD concentration, $6 \mu \mathrm{g} \mathrm{CdSe} / \mathrm{ZnS}$ QDs per mouse) for 0.5 or $2.5 \mathrm{~h}$. The EMT-6 tumor-bearing mice treated with saline 
was carried out as control. Before fluorescence imaging, the mice were anesthetized by intraperitoneal injection of sodium pentobarbital $(50 \mathrm{mg} / \mathrm{kg})$. Then the fluorescence imaging of tumor-bearing nude mice was performed by using IVIS ${ }^{\circledR}$ Lumina Series III (PerkinElmer, Inc., Waltham, MA, USA). The in vitro representative fluorescence images of L-PLGA/ PPF samples with different concentrations in 96-well plate were imaged under a chemilluminescent and fluorescent imaging system.

\section{In vivo antitumor effects}

The EMT-6 tumor-bearing mice were randomly divided into the following five groups treated with different formulations (n=6 per group): 1) saline (control); 2) non-DOX-loaded luminescent/magnetic PLGA-based hybrid nanoparticles (LM-PLGA/PPF); 3) non-DOX-loaded luminescent/ magnetic PLGA-based hybrid nanoparticles with VEGF shRNA (LM-PLGA/PPF/VEGF shRNA) (0.48 mg/kg VEGF shRNA); 4) PLGA nanoparticles coloaded with QDs, DOX, and $\mathrm{Fe}_{3} \mathrm{O}_{4}$ modified with PPF conjugate and $\mathrm{SC}$ shRNA(LDMPLGA/PPF/SC shRNA) (4 mg/kg DOX and $0.48 \mathrm{mg} / \mathrm{kg}$ SC shRNA); and 5) PLGA nanoparticles coloaded with QDs, DOX, and $\mathrm{Fe}_{3} \mathrm{O}_{4}$ modified with PPF conjugate and VEGF shRNA (LDM-PLGA/PPF/VEGF shRNA) (4 mg/kg DOX and $0.48 \mathrm{mg} / \mathrm{kg}$ VEGF shRNA). Mice in each group were administered an intratumoral injection on days 0,6 , 12 , and 18 . Subsequently, the tumor sizes and body weights were recorded every 3 days for a period of 3 weeks using a Vernier caliper and electronic weighing scale. After the last measurement, all mice were sacrificed/euthanized, and the tumor xenografts were excised, weighed, and photographed. The dissected tumors and major organs, including the heart, liver, spleen, lungs, and kidneys, were gathered and then fixed in a $4 \%(\mathrm{w} / \mathrm{v})$ paraformaldehyde solution for the following histological examinations that involved H\&E staining and terminal deoxynucleotidyl transferase dUTP nick-end labeling (TUNEL) assays, which were performed for the evaluation of antitumor effects.

\section{Statistical analysis}

All experiments were carried out at least in triplicate. Data were presented as the mean \pm SD. Statistical analysis was performed using GraphPad Prism Software version 6.0 (GraphPad Software Inc., San Diego, CA, USA), and the statistical significances were determined by using the Student's $t$-test. Differences were considered to be significant at $P$-value $<0.05$.

\section{Results and discussion Synthesis and characterization of PPF}

The composition and chemical structure of the resulting FA-PEG-COOH and PPF conjugates were determined by ${ }^{1} \mathrm{H}-\mathrm{NMR}$. In the spectra obtained from PEG-FA in deuterated DMSO (DMSO- $d_{6}$ ) and PPF in $\mathrm{D}_{2} \mathrm{O}$ (Figure S1), the chemical shift observed at $3.50 \mathrm{ppm}$ was attributed to the $-\mathrm{CH}_{2}-$ proton peak of PEG, and those at 6.62, 7.61, and $8.62 \mathrm{ppm}$ were attributed to $\mathrm{H}$ in the FA benzene ring, clearly indicating that FA has been successfully conjugated to $\mathrm{NH}_{2}$-PEG-COOH. Meanwhile, the chemical shifts at 2.40-3.70 ppm were assigned to the $-\mathrm{CH}_{2}-$ proton peaks of PEI, suggesting that FA-PEG-COOH was conjugated onto the PEI molecular chain. ${ }^{11,51}$

\section{Synthesis and characterization of LDM- PLGA/PPF/VEGF shRNA nanocomposites}

The FA-targeted PEGylated PLGA nanocomposites were fabricated by combining a double emulsion method and covalent linkage. Figure 2A shows the SEM images of LDMPLGA, LDM-PLGA/PPF, and LDM-PLGA/PPF/VEGF shRNA, and the TEM image of LDM-PLGA. SEM images showed that the as-synthesized nanoparticles were dispersed and had a regular spherical morphology, $\sim 300 \mathrm{~nm}$ in size (Figure 2Aa-c). PPF modification and VEGF shRNA complexation did not have an effect on nanoparticle morphology and showed negligible aggregation in aqueous solution, likely due to the increased surface charge of the nanocomposites and VEGF shRNA electrostatic interactions. TEM images of LDM-PLGA (Figure 2Ad) indicated that $\mathrm{Fe}_{3} \mathrm{O}_{4}$ nanoparticles were scattered around the PLGA matrix, suggesting that the magnetic nanocrystals were successfully embedded within the nanoparticles. The mean diameters of the LDM-PLGA (Figure 2Be) and LDM-PLGA/PPF (Figure 2Bf) nanoparticles dispersed in aqueous solution were 327 and $336 \mathrm{~nm}$, although their overall size increased slightly following surface modification by PPF. Additionally, the surface potential of LDM-PLGA/PPF after modification was positive charged $(+17.2 \mathrm{mV})$ compared with the negative charge of LDMPLGA (-32.0 mV) (Figure 2C), thus allowing increased loading of negatively charged VEGF shRNA by electronic absorption. The changes in zeta potential suggest the successful modification of PPF and VEGF shRNA absorption. As shown in Figure 3A, thermogravimetric analysis was carried out to quantify the overall mass loss of the various coated polymers under high temperature. The residual weights 

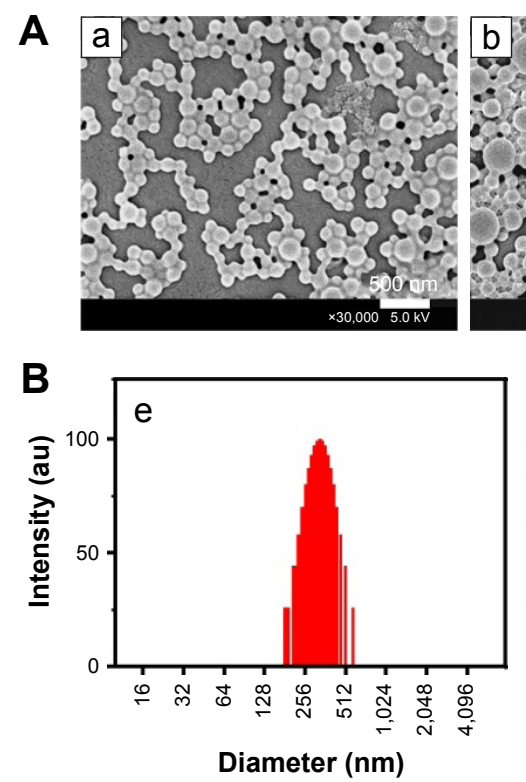
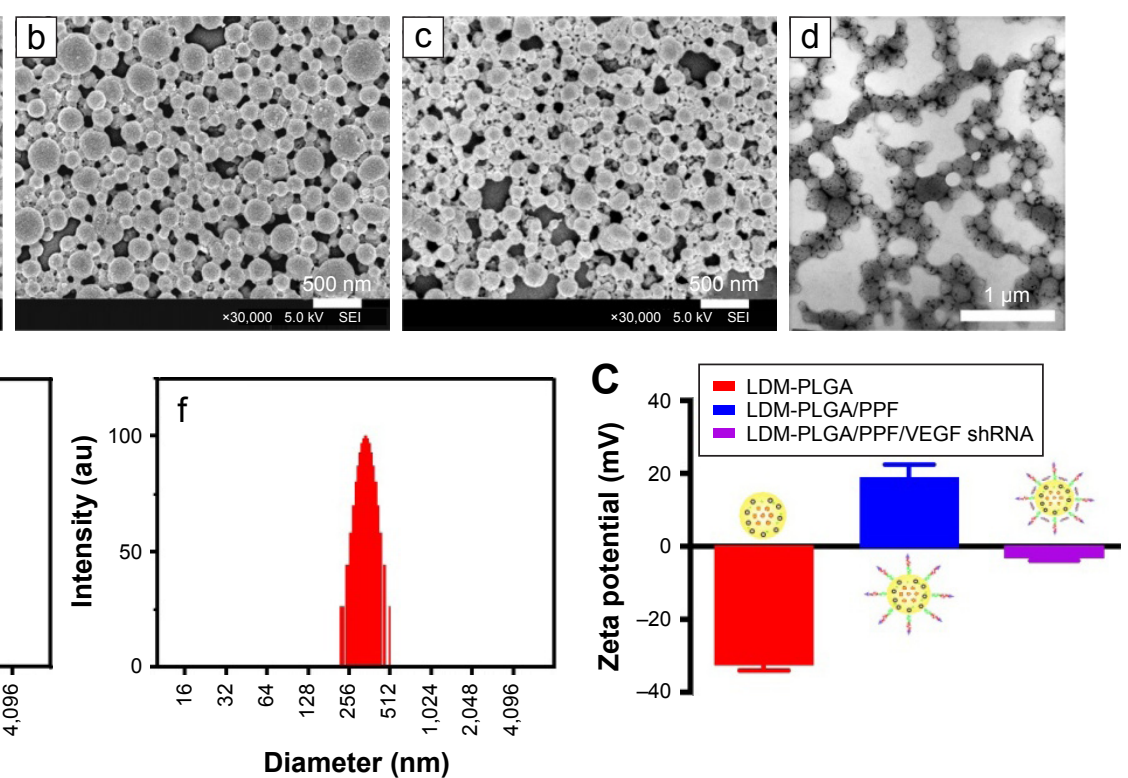

Figure 2 Characterization of the as-synthesized nanoparticles.

Notes: (A) Morphology: (a) Scanning electron microscopy images of the PLGA nanoparticles coloaded with QDs, DOX, and Fe $\mathrm{O}_{4}$ without modification (LDM-PLGA); (b) PLGA nanoparticles coloaded with QDs, DOX, and $\mathrm{Fe}_{3} \mathrm{O}_{4}$ modified with PPF conjugate (LDM-PLGA/PPF); (c) PLGA nanoparticles coloaded with QDs, DOX, and $\mathrm{Fe}_{3} \mathrm{O}_{4}$ modified with PPF conjugate and VEGF shRNA (LDM-PLGA/PPF/VEGF shRNA); (d) transmission electron microscopy images of LDM-PLGA. (B) The particle size distributions of (e) LDM-PLGA and (f) LDM-PLGA/PPF, determined by dynamic light scattering. (C) Zeta potentials of LDM-PLGA, LDM-PLGA/PPF, and LDM-PLGA/PPF/ VEGF shRNA nanoparticle composites in PBS $(\mathrm{pH} 7.4)$ at room temperature $\left(25^{\circ} \mathrm{C}\right)$.

Abbreviations: DOX, doxorubicin; PPF, PEI-PEG-FA; PEI-PEG-FA, polyethyleneimine premodified with polyethylene glycol-folic acid; QD, quantum dot; shRNA, small hairpin RNA; VEGF, vascular endothelial growth factor.

of LDM-PLGA and LDM-PLGA/PPF nanoparticles were $9.55 \%$ and $2.53 \%$, respectively, at $\sim 500^{\circ} \mathrm{C}$. Therefore, the amount of PPF polymer coating the LDM-PLGA surface was calculated to be $7.02 \%$. Agarose gel electrophoresis
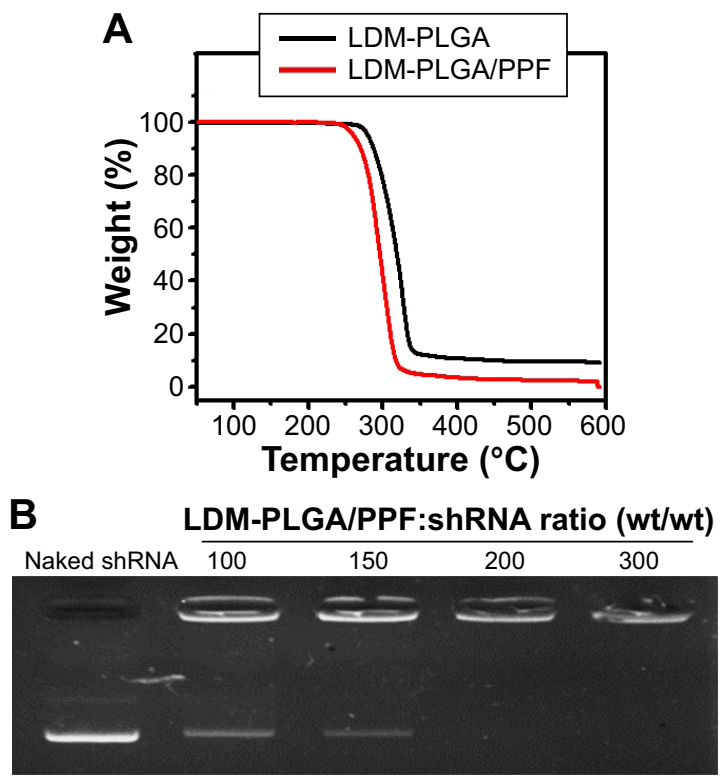

Figure 3 (A) Thermogravimetric analysis of LDM-PLGA and LDM-PLGA/PPF. (B) Agarose gel electrophoresis assay of LDM-PLGA/PPF/VEGF shRNA at various weight ratios of LDM-PLGA/PPF to VEGF shRNA.

Abbreviations: PPF, PEI-PEG-FA; PEI-PEG-FA, polyethyleneimine premodified with polyethylene glycol-folic acid; shRNA, small hairpin RNA; VEGF, vascular endothelial growth factor. was performed to further analyze the binding capacity of LDM-PLGA/PPF to VEGF shRNA (Figure 3B), which was shown to increase gradually from a 100:1 weight ratio to $300: 1$. When the weight ratio was more than 150:1, most of the VEGF shRNA was absorbed onto the LDM-PLGA/ PPF nanoparticles.

Successful encapsulation of DOX into LDM-PLGA was confirmed by the UV-vis absorbance spectra of the samples (Figure 4Aa). DOX-loaded PLGA nanoparticles exhibited characteristic DOX absorption peaks at $480 \mathrm{~nm}$, such as those of free DOX. In addition, the fluorescence emission peaks of DOX-loaded nanoparticles were centered at 550 and $590 \mathrm{~nm}$ when excited at $480 \mathrm{~nm}$, similar to free DOX (Figure 4Ab). These results demonstrate the successful encapsulation of DOX within the nanoparticles. Encapsulation efficiency was characterized as the weight percentage of DOX incorporated into LDM-PLGA/PPF. When the initial loading concentration was $60 \mathrm{mg}$ DOX per gram of PLGA, the encapsulation efficiency was $\sim 62.97 \%$. The amount of DOX released from LDM-PLGA or LDM-PLGA/PPF was assessed under two $\mathrm{pH}$ conditions (PBS solution $\mathrm{pH} 7.4$ or acetate buffer solution $\mathrm{pH}$ 4.4) representing in vivo physiological conditions and the endosomal/lysosomal microenvironment, respectively. The DOX release profiles of LDM-PLGA are shown in Figure S2A. The amount of DOX released was higher at 
A a

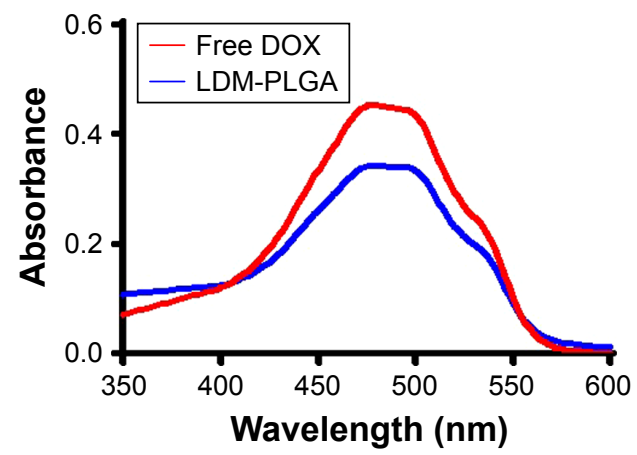

B c

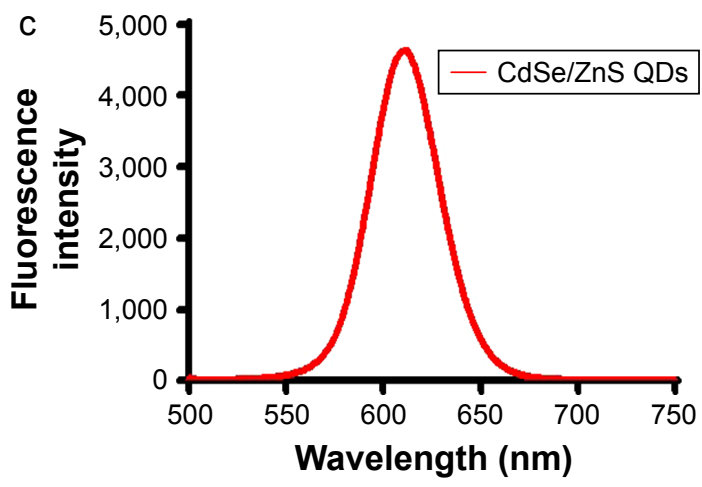

C

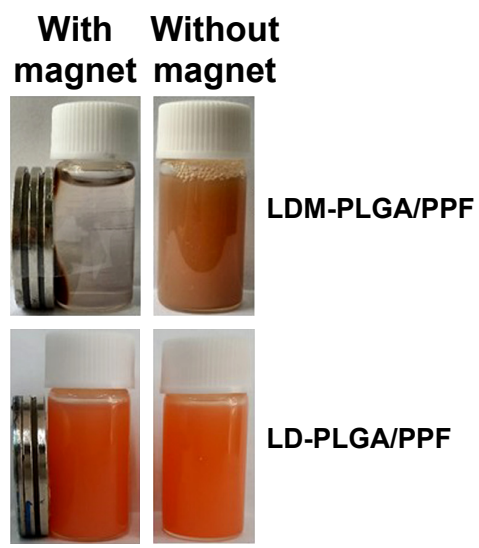

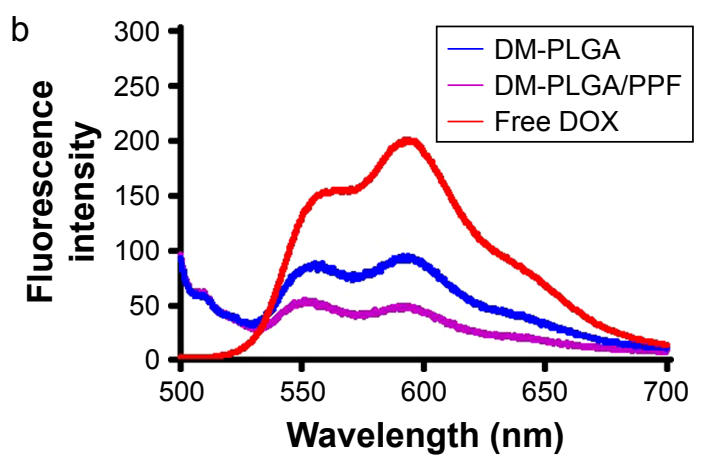

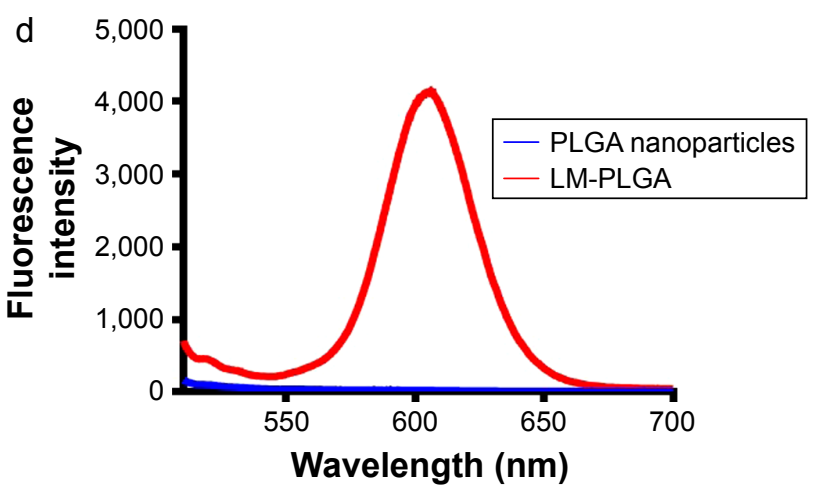

D

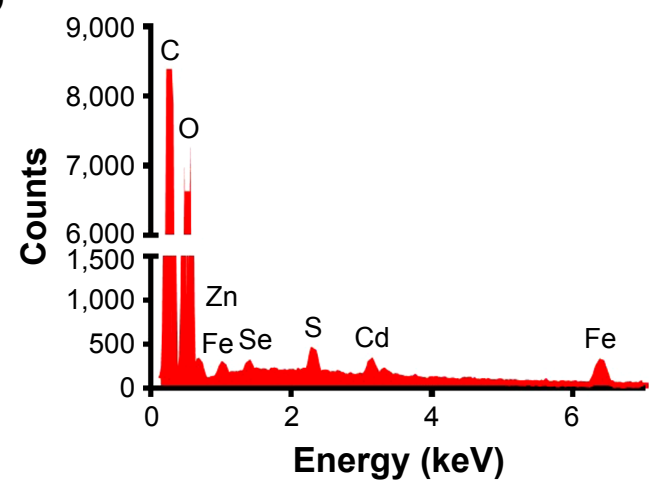

Figure 4 UV-vis and photoluminescent characterization.

Notes: (A) UV-vis absorption (a) and photoluminescence spectra (b) of free DOX and the as-prepared nanoparticles. (B) Photoluminescence spectra of CdSe/ZnS QDs (c) and LM-PLGA (d) (excitation wavelength $490 \mathrm{~nm}$ ). (C) Photographs of LDM-PLGA/PPF and LD-PLGA/PPF with or without an applied magnetic field. (D) Elemental analysis of LDM-PLGA nanoparticles.

Abbreviations: DOX, doxorubicin; PLGA, poly(D,L-lactic-co-glycolic acid); PPF, PEI-PEG-FA; PEI-PEG-FA, polyethyleneimine premodified with polyethylene glycol-folic acid; QDs, quantum dots.

pH 4.4 than at $\mathrm{pH} 7.4$ and showed a burst release behavior during the first $10 \mathrm{~h}$, followed by sustained release in the remaining assay time. Approximately $47.33 \%$ and $44.72 \%$ of DOX were released within $48 \mathrm{~h}$ from LDM-PLGA at $\mathrm{pH}$ 4.4 and 7.4, respectively. Nevertheless, LDM-PLGA/PPF exhibited only minor ( $21.52 \%)$ DOX release within $120 \mathrm{~h}$, even at $\mathrm{pH} 4.4$ (Figure S2B). The modification conjugate PPF prevents drug leakage from the PLGA backbone and hinders the DOX molecules from rapidly diffusing into the outer aqueous solution within a short time. Additionally, it hinders the penetration of water into the inner nanoparticles compared with the nonmodified nanoparticles (LDM-PLGA); this may reduce the nanoparticle degradation rate and increase their stability. ${ }^{52}$ Therefore, the modified nanoparticles may have an enhanced chemotherapeutic effect through minimal drug leakage in the blood and an accumulation of LDM-PLGA/ PPF, as well as the continuous release of DOX in tumor tissues. The $\mathrm{pH}$-responsive release of DOX was attributed to the protonation of the amine groups on the DOX molecule in an acidic environment, which increases the hydrophilicity of DOX under acidic $\mathrm{pH}$ conditions, encouraging its release. ${ }^{53,54}$ 
Figure 4B shows the photoluminescence spectra of CdSe/ZnS QDs, blank PLGA nanoparticles, and LMPLGA nanoparticles. The fluorescence emission peak of $\mathrm{CdSe} / \mathrm{ZnS}$ QDs is centered at $605 \mathrm{~nm}$ when excited at $490 \mathrm{~nm}$ (Figure 4Bc). Further, LM-PLGA shows an emission peak at $605 \mathrm{~nm}$ when excited using the optimum wavelength of $490 \mathrm{~nm}$ (Figure 4Bd), demonstrating the successful incorporation of CdSe/ZnS QDs into the PLGA nanoparticles, thus endowing them with photoluminescent properties.

The magnetic characteristics of LDM-PLGA/PPF were assessed by placing a permanent magnet near the dispersed nanoparticle aqueous solution (Figure 4C). LDM-PLGA/ PPF nanoparticles were attracted toward the magnet, whereas the nonmagnetic PLGA nanoparticles (LD-PLGA/PPF) were not, thus showing the excellent magnetic responsiveness of LDM-PLGA/PPF. These properties may allow specific targeting of the area with drugs under an external magnetic field. The energy dispersive X-ray analysis (Figure 4D) of LDM-PLGA confirmed the existence of $\mathrm{C}, \mathrm{O}, \mathrm{Fe}, \mathrm{Zn}, \mathrm{S}$, $\mathrm{Cd}$, and Se elements, indicating the successful incorporation of $\mathrm{CdSe} / \mathrm{ZnS}$ QDs, DOX, and $\mathrm{Fe}_{3} \mathrm{O}_{4}$ into the nanoparticles and further demonstrating the successful preparation of hybrid nanoparticles.
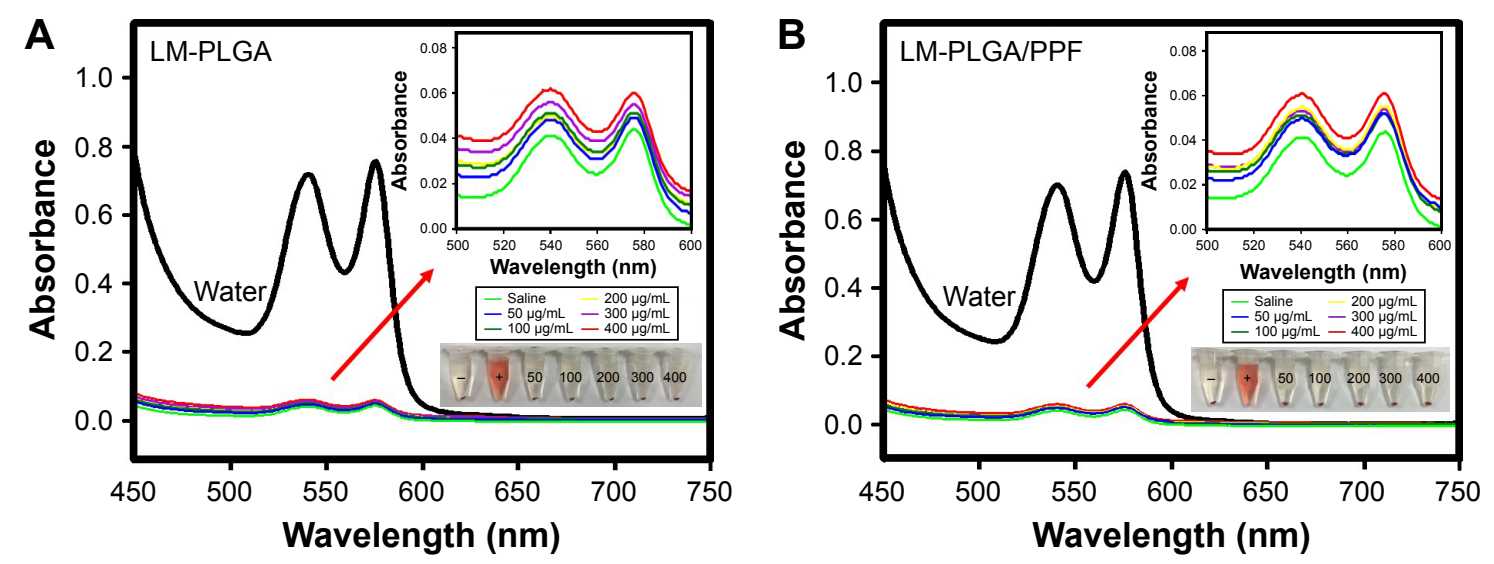

C

Saline
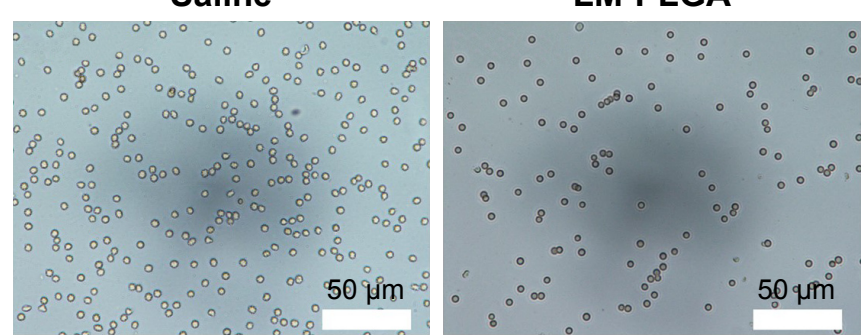

LM-PLGA

LM-PLGA/PPF

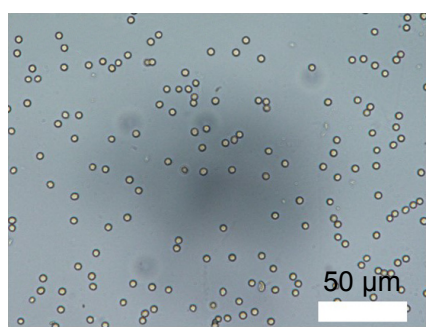

Figure 5 In vitro hemolysis assay. Hemolytic activity of the LM-PLGA (A) and LM-PLGA/PPF (B) at various nanocomposite concentrations (50, I00, 200, 300, and 400 $\mu \mathrm{g} / \mathrm{mL}$, respectively), incubated with rat red blood cells at $37^{\circ} \mathrm{C}$ for $2 \mathrm{~h}$. Saline and water were used as negative and positive controls, respectively. Insets on the top right are the enlarged UV-vis spectra and bottom-right insets show the photograph of red blood cells treated with LM-PLGA and LM-PLGA/PPF at various concentrations. (C) Optical microscopy images of the dispersion of erythrocytes treated with different nanoparticles. Saline was used as a control.

Abbreviations: PLGA, poly(D,L-lactic-co-glycolic acid); PPF, PEI-PEG-FA; PEI-PEG-FA, polyethyleneimine premodified with polyethylene glycol-folic acid. 
dispersibility and no obvious aggregation (Figure 5C), indicating that their excellent hemocompatibility makes them suitable for successful systemic administration.

Nanoparticle stability is also significant for further pharmaceutical applications. Herein, the dispersibility of LDM-PLGA/PPF in PBS, saline, and Roswell Park Memorial Institute 1640 cell culture medium with and without serum was evaluated, as were the changes in hydrodynamic size and zeta potential in PBS. As shown in Figure S3, the nanoparticles were well dispersed in different aqueous media, and no significant changes in hydrodynamic size and zeta potential were observed for up to 2 weeks, suggesting their good stability. Thus, the fabricated nanocomposites displayed excellent biocompatibility and could be used as a drug delivery system for various nanomedicine applications.

\section{In vitro cytotoxicity}

It is essential to evaluate the potential of LM-PLGA/PPF nanoparticles as a safe and effective delivery system and to determine the safe concentration for siRNA and drug delivery. ${ }^{56,57}$ The MTS assay was performed to investigate the in vitro cytotoxicity on HUVECs (normal cells) and HeLa cells (cancerous cells) at various nanoparticle concentrations for 48 and $72 \mathrm{~h}$. LM-PLGA/PPF nanoparticles did not show any apparent cytotoxicity when incubated with HeLa cells or HUVECs using the studied concentration range $(0-250 \mu \mathrm{g} / \mathrm{mL})$ for $48 \mathrm{~h}$ (Figure S4A), and the cell viability was $>90 \%$. With an increase in incubation time to $72 \mathrm{~h}, \mathrm{LM}-\mathrm{PLGA} / \mathrm{PPF}$ nanoparticles exhibited minor cytotoxicity at concentrations $>150 \mu \mathrm{g} / \mathrm{mL}$, especially on HUVECs (Figure S4B), likely due to the highly positively charged potential of PPF conjugates coated on PLGA particles at high concentrations, leading to strong electrostatic interactions with the negatively charged cell membranes. ${ }^{12,58}$ These results indicate that, despite the slight cytotoxicity due to increased incubation time and high concentrations, LMPLGA/PPF nanoparticles possess favorable biocompatibility when incubated with cells for $48 \mathrm{~h}$.

To further determine the therapeutic efficacy of DOXloaded nanoparticles (LDM-PLGA/PPF), HeLa and EMT-6 cells were incubated with different drug formulations under various DOX concentrations for $48 \mathrm{~h}$, followed by quantification of cell viability. Non-DOX-loaded nanoparticles showed no cytotoxicity at the studied concentration range (Figure 6A), suggesting an excellent cytocompatibility as nanocarriers. Free DOX and DOX-loaded nanoparticles exhibited a dose-dependent increase in cytotoxicity; of note, LDM-PLGA/PPF displayed higher cytotoxicity than
A \begin{tabular}{|ll|}
\hline - Control & $\square$ LM-PLGA/PPF \\
$\square$ LM-PLGA/PPF + FA & $\square$ LDM-PLGA/PPF \\
$\square$ LDM-PLGA/PPF + FA & $\square$ Free DOX \\
\hline
\end{tabular}

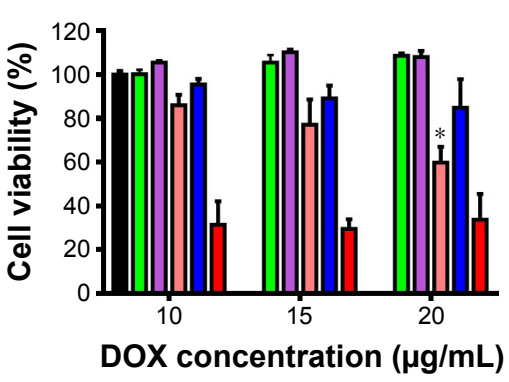

D Control

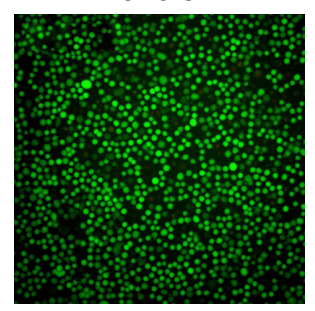

B
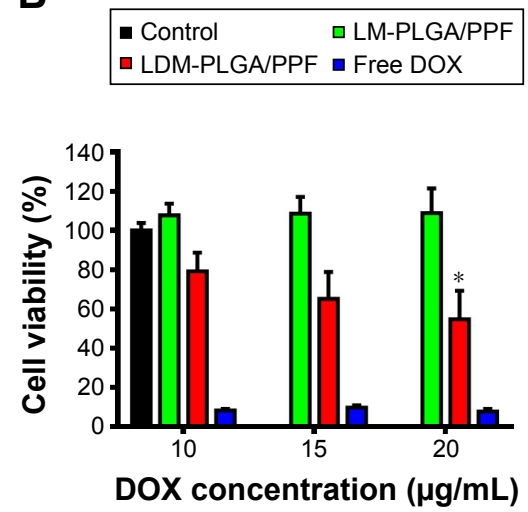

PLGA/PPF

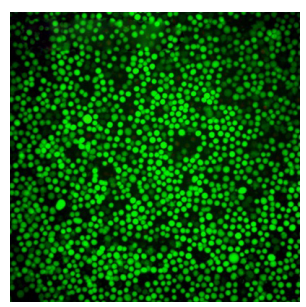

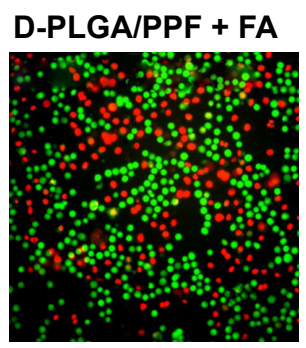

C

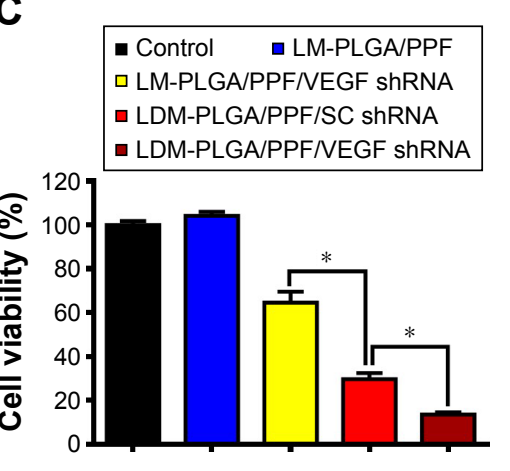

D-PLGA/PPF

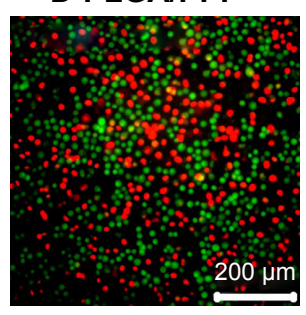

Figure 6 In vitro cell viability of HeLa cells $(\mathbf{A})$ and EMT-6 cells (B) treated with free DOX, LDM-PLGA/PPF, and LM-PLGA/PPF with different DOX concentrations for $48 \mathrm{~h}$ with or without preincubation with free folic acid (1.25 mM). (C) Synergistic therapy efficacy of LDM-PLGA/PPF/VEGF shRNA nanocomposites against EMT-6 cells at $25 \mu \mathrm{g} / \mathrm{mL}$ of DOX concentration for $72 \mathrm{~h}$. (D) Fluorescence microscopy images of calcein-AM and propidium iodide costaining of HeLa cells after various treatments at a DOX concentration of $20 \mu \mathrm{g} / \mathrm{mL}$. Green and red colors represent live and dead cells, respectively. $* P<0.05$.

Abbreviations: DOX, doxorubicin; PLGA, poly(D,L-lactic-co-glycolic acid); PPF, PEI-PEG-FA; PEI-PEG-FA, polyethyleneimine premodified with polyethylene glycol-folic acid; shRNA, small hairpin RNA; VEGF, vascular endothelial growth factor. 
that of HeLa cells pretreated with free FA. When the DOX concentration was $20 \mu \mathrm{g} / \mathrm{mL}$, the cell viability of HeLa cells treated with LDM-PLGA/PPF was only 59\% without preincubation with free FA. These results may be attributed to the fact that FA-conjugated nanoparticles was favorable for tumor cells uptake via ligand-receptor interactions when there is no free FA to competitively inhibit this, leading to an increased delivery of DOX into the HeLa cells. ${ }^{47}$ Furthermore, free DOX showed a higher cytotoxicity compared to any of the DOX-loaded nanocomposites at the same DOX dosages, which is in agreement with previous reports. ${ }^{4,54,59,60}$ This effect is mainly attributed to the slow and continuous drug release properties (Figure S2B) and to the longer time required for DOX to enter the cell nucleus compared with free DOX, thus leading to an accumulation of DOX in tumor cells and prolonged (long-term) cytotoxicity. This result also confirmed the possibility that the long-term and accumulated drug release of as-presented drug-loaded nanocomposites can reduce the side effects of DOX on the unexpected positions in in vivo applications compared with free DOX and enhance cancer chemotherapy effects.

The cytotoxicity of various drug formulations incubated with EMT-6 cells was also evaluated (Figure 6B). Likewise, the increase in DOX concentration resulted in lower cell viability, by $\sim 54 \%$, when the DOX concentration reached $20 \mu \mathrm{g} / \mathrm{mL}$. The viability of EMT-6 cells after incubation with LDM-PLGA/PPF/VEGF shRNA nanocomposites for $72 \mathrm{~h}$ for siRNA-based cancer therapy was only 14\% (Figure 6C) and was much lower than that of cells treated with other drug formulations at the same DOX dosage $(25 \mu \mathrm{g} / \mathrm{mL} ; P<0.05)$. VEGF shRNA (300 ng) was incubated with LDM-PLGA/ PPF for $30 \mathrm{~min}$ prior to addition to the 96-well plates for cell treatment. Cells treated with the DOX/VEGF shRNA codelivery nanocarrier also exhibited a significant antiproliferation effect, whereas the monotherapy group (LMPLGA/PPF/VEGF shRNA) resulted in nonsignificant inhibition on cell proliferation.

In order to visually evaluate the therapeutic effects of D-PLGA/PPF in vitro, fluorescence images of HeLa cells costained with calcein-AM (green, live cells) and PI (red, dead cells) were carried out to qualitatively identify live and dead cells, respectively. HeLa cells treated with D-PLGA/PPF (DOX $20 \mu \mathrm{g} / \mathrm{mL}$ ) for $6 \mathrm{~h}$ without preincubation with free FA to competitively inhibit the ligand-receptor interaction showed significantly more dead cells compared to control treatment (Figure 6D). Thus, fluorescence imaging results were in agreement with those of the cytotoxicity assay.

\section{Magnetic and folate receptor dual targeting in vitro}

We further determined the physicochemical targeting effects from $\mathrm{Fe}_{3} \mathrm{O}_{4}$ and active targeting from folate of LDM-PLGA/ PPF in cell culture experiments. HeLa cells treated with LDM-PLGA/PPF in a cell culture dish were exposed to an external $0.42 \mathrm{~T} \mathrm{Nd-Fe-B} \mathrm{magnet} \mathrm{(Figure} \mathrm{7Aa).} \mathrm{After}$ 6-h incubation, cells were washed three times, and the fluorescence images were obtained by inverted fluorescence microscopy at different locations in the culture dish, resulting in a decline of the intracellular DOX fluorescence signal with increasing distance from the magnet. Similar results were observed when the cells were pretreated with free folate prior to culturing with LDM-PLGA/PPF nanoparticles (Figure 7Ab). However, fluorescence signals were reduced to a certain extent compared to those without free folate pretreatment, suggesting that the folate receptor-mediated cell targeting effect was more obvious when the cells were far away from the magnetic field. The biological targeting effect via folate was further demonstrated with the ligand competing assay (Figure 7B), in which HeLa cells were pretreated with free folate prior to culturing with the FAconjugated nanoparticles. A reduction in DOX fluorescence signal was observed compared with cells without free folate pretreatment, indicating a decreased uptake of nanoparticles by HeLa cells. Quantitative fluorescence intensity results shown in Figure $7 \mathrm{C}$ were consistent with those of fluorescence microscopy. These results thus demonstrated that the uptake of LDM-PLGA/PPF was mediated by the folate receptor on the cell membrane. ${ }^{61,62}$ Taken together, the above data indicate that LDM-PLGA/PPF nanoparticles could serve as an effective carrier with magnetic and FA dual targeting effects.

\section{Cellular uptake and endosomal/lysosomal escape}

The cellular uptake and intracellular drug release behavior of free DOX, DM-PLGA, and DM-PLGA/PPF were confirmed by confocal microscopy following incubation with HeLa cells for $6 \mathrm{~h}$. As shown in Figure 8A, a red fluorescence concentration can be observed in the cell nuclei after incubation of HeLa cells with free DOX, showing that free DOX is mainly distributed in the nucleus after cellular uptake and suggesting an excellent permeability and uptake of DOX into the cells. Additionally, the DOX delivery behavior of DM-PLGA was similar to that of free DOX as characterized by its intensive red fluorescence in the nucleus. This result is due to the drug release profile of DM-PLGA (Figure S2A), 

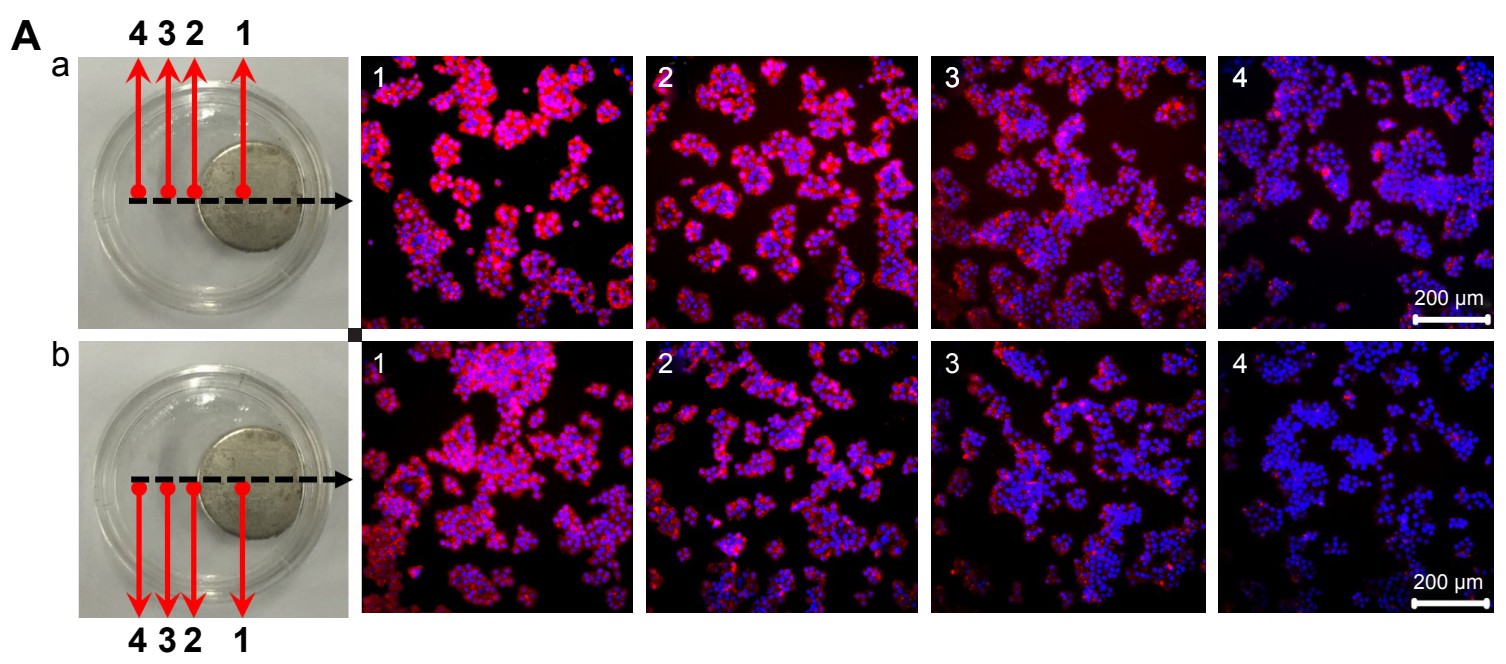

4321
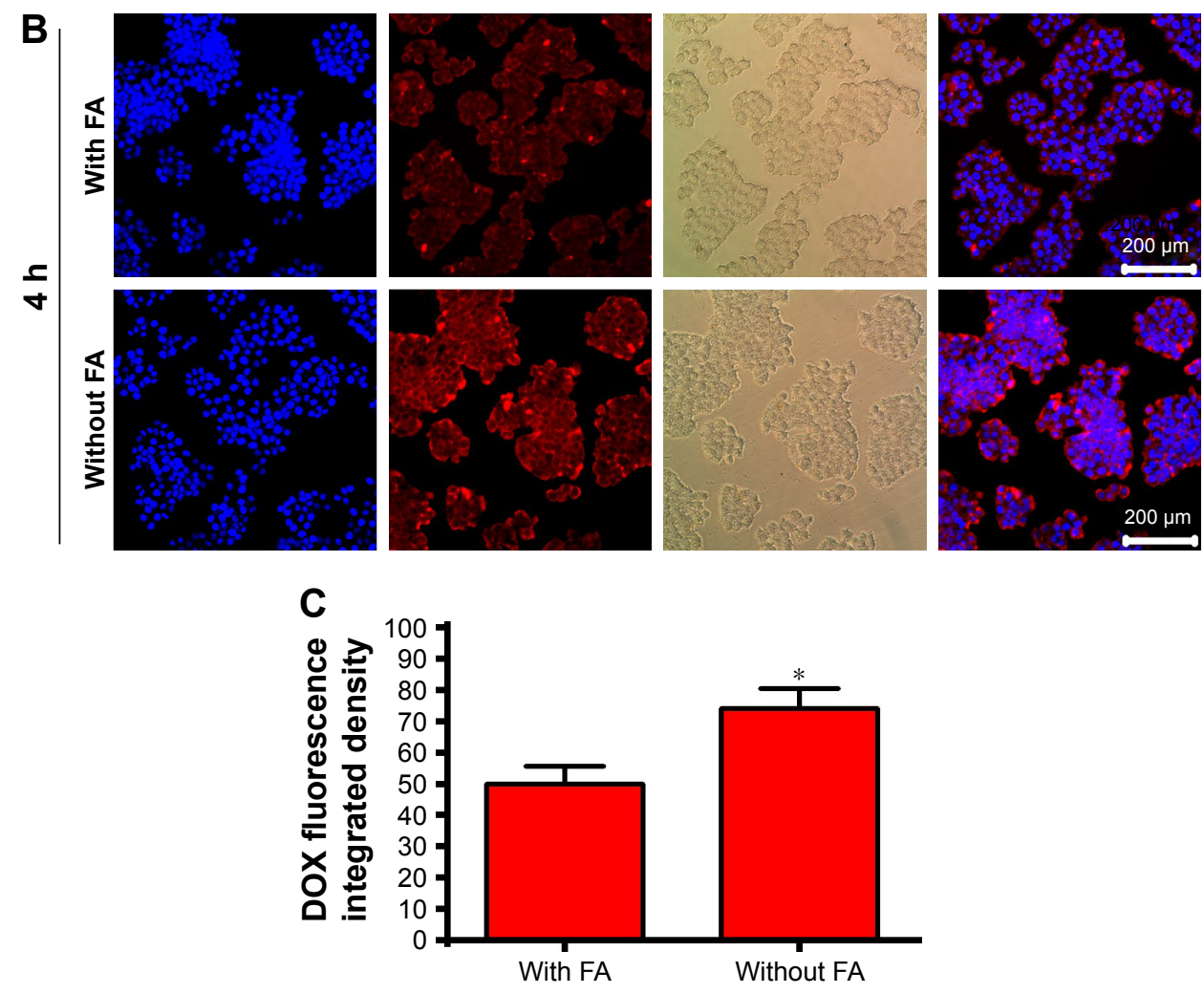

Figure 7 The fluorescence disparity between the samples demonstrated the dynamic interplay of magnetic targeting versus biological targeting via folate under different magnetic fields.

Notes: (A) Fluorescence microscopy images of LDM-PLGA/PPF nanoparticles treated with HeLa cells (a) without and (b) with preincubation with free FA (I.25 mM) before exposure to an external magnetic field for $8 \mathrm{~h}$. (B) Fluorescence microscopy images of HeLa cells with or without preincubation with free FA (I.25 mM) before treatment with LDM-PLGA/PPF nanoparticles for $4 \mathrm{~h}$. (C) The intracellular DOX was quantitatively detected by fluorescence spectrophotometry. ${ }^{* P}<0.05$ compared with the FA-treated group.

Abbreviations: DOX, doxorubicin; FA, folic acid; PLGA, poly(D,L-lactic-co-glycolic acid); PPF, PEI-PEG-FA; PEI-PEG-FA, polyethyleneimine premodified with polyethylene glycol-folic acid.

wherein DOX is burst released for cellular uptake from DM-PLGA in an acid plasma environment, which is able to easily and rapidly enter into the nucleus. However, DMPLGA/PPF significantly enhanced the delivery of DOX to the cells, as observed by an increase in red DOX fluorescence in the cytoplasm. This result is also consistent with the drug release profile of DM-PLGA/PPF (Figure S2B), wherein the continuously released DOX was able to accumulate in tumor cells, subsequently entering the nucleus. Thus, the as-prepared DM-PLGA/PPF nanoparticles can be first endocytosed into the cells, followed by a subsequent continuous release of DOX in an acid cytoplasm environment 
A
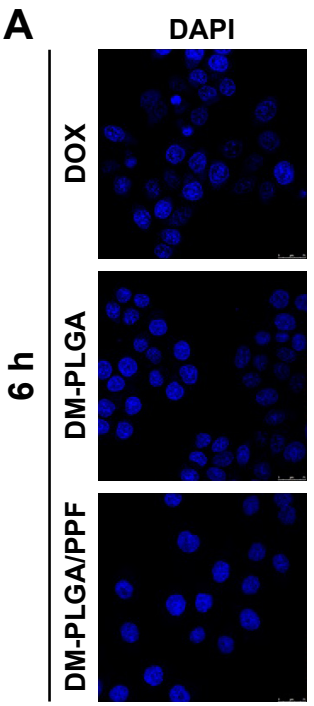

DOX
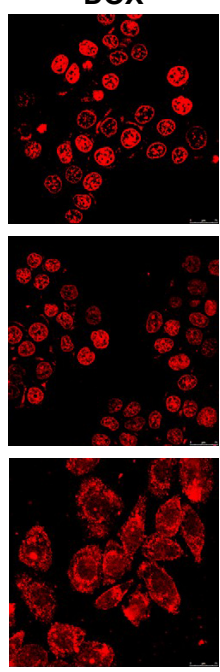

Merge
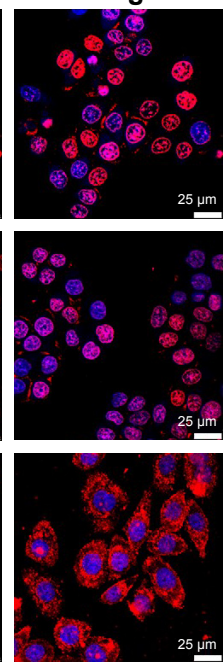

\section{B Bright field}
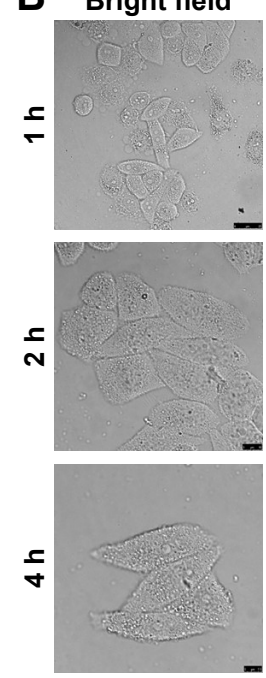

DOX
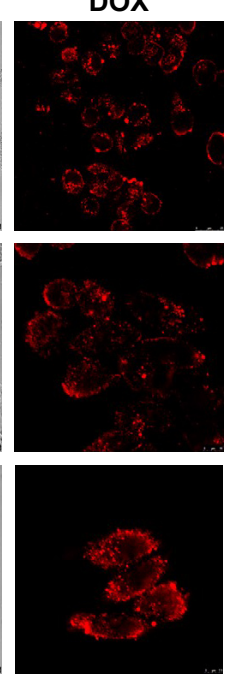
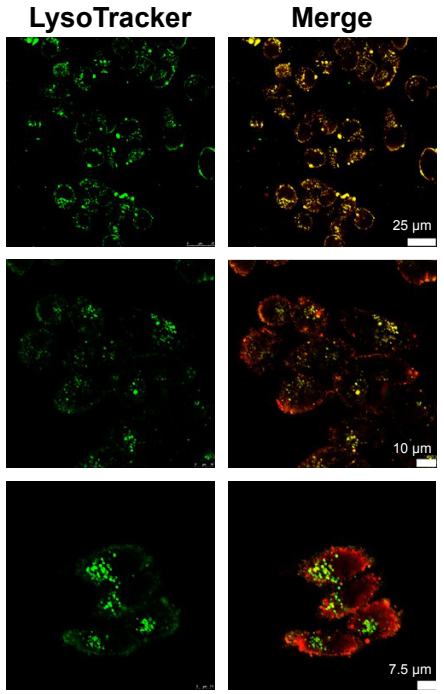

Figure 8 Cellular uptake and endosomal/lysosomal escape of nanocomposites by confocal laser scanning microscopy.

Notes: (A) HeLa cells were treated with free DOX, DM-PLGA, and DM-PLGA/PPF for 6 h. (B) Endosomal/lysosomal escape of DM-PLGA/PPF nanoparticles incubated with HeLa cells at a DOX concentration of $5 \mu \mathrm{g} / \mathrm{mL}$ and $37^{\circ} \mathrm{C}$ for various time intervals.

Abbreviations: DOX, doxorubicin; DAPI, 4,6-diamino-2-phenylindole; PLGA, poly(D,L-lactic-co-glycolic acid); PPF, PEI-PEG-FA; PEI-PEG-FA, polyethyleneimine premodified with polyethylene glycol-folic acid.

and entrance into the cell nucleus, leading to a slower and prolonged DOX accumulation in the nucleus. ${ }^{63}$

To further track the intracellular distribution of DMPLGA/PPF nanoparticles following cellular uptake, HeLa cells were treated with DM-PLGA/PPF for 1, 2, or $4 \mathrm{~h}$, and individually stained with LysoTracker Green to label the nucleus and lysosomes. As shown in Figure 8B, an overlay of the green (LysoTracker Green-stained lysosome) and red (DOX) fluorescence after $1 \mathrm{~h}$ of incubation revealed that the DM-PLGA/PPF nanoparticles were localized in lysosomes. When the incubation time was increased to $2 \mathrm{~h}$, a small amount of released DOX from the nanoparticles was gradually released from the lysosomes to the cytoplasm, as observed by a decrease in the colocalization signals of the merged image. After $4 \mathrm{~h}$ incubation, the red fluorescence of DM-PLGA/PPF exhibited minimal colocalization with green fluorescence of lysosomes, as shown by the separated green and red fluorescence, suggesting that the majority of the DM-PLGA/PPF nanoparticles or released DOX escaped from the lysosomes to the cytoplasm. Significantly stronger yellow fluorescent co-localization signals observed in HeLa cells substantiated the cellular uptake of DM-PLGA/PPF by endocytosis and distribution into the endo/lysosomal compartments. ${ }^{64,65}$

\section{$M R$ and animal fluorescence imaging studies}

$\mathrm{Fe}_{3} \mathrm{O}_{4}$ nanoparticles have been proven to be excellent contrast agents in $T_{2}$-weighted MR imaging. Therefore, to validate the potential of LDM-PLGA/PPF nanoparticles as an MR contrast agent for image-guided therapy, HeLa cells were cultured with LDM-PLGA/PPF nanoparticles at different Fe concentrations $(1.8,2.3,3.4,4.6$, and $6.8 \mu \mathrm{g} / \mathrm{mL})$ for $12 \mathrm{~h}$. $\mathrm{T}_{2}$-weighted MR phantom images of the cells were obtained (Figure 9A), whereby, with the increasing Fe concentrations during the incubation, the MR signal intensity was seen to gradually decrease (signal darkening). At high Fe concentrations ( 4.6 and $6.8 \mu \mathrm{g} / \mathrm{mL}$ ), a significantly decreased MR signal intensity was observed, and the $T_{2}$-weighted MR images were darker than those of cells treated with low Fe concentrations, indicating that LDM-PLGA/PPF showed a significant negative contrast enhancement labeled with cells. Quantitative analysis results showed that MR signal intensity decreased with the Fe concentration in HeLa cells (Figure 9B), which was also consistent with the $T_{2}$-weighted MR images. The in vivo $T_{2}$-weighted MR imaging for EMT-6 tumorbearing mice after intratumoral injection of LDM-PLGA/PPF for $24 \mathrm{~h}$ (Figure 9C) showed that, compared with the control group without injection (left image), an enhanced decrease in MR signal (enhanced darkening) was observed in the tumor site after injection of $\mathrm{Fe}_{3} \mathrm{O}_{4}$-loaded PLGA nanoparticles (right image). Taken together, these data demonstrate that the as-prepared nanoparticles could be used as a probe for $T_{2}$-weighted MR imaging for cancer diagnosis.

The representative fluorescence images of $\mathrm{CdSe} / \mathrm{ZnS}$ QDs were confirmed by using the chemilluminescent and fluorescent imaging system (Figure S5). The fluorescence 
A

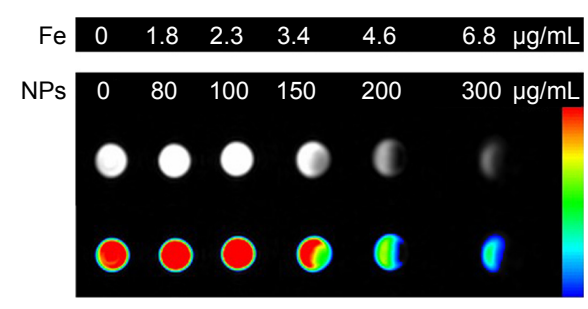

B

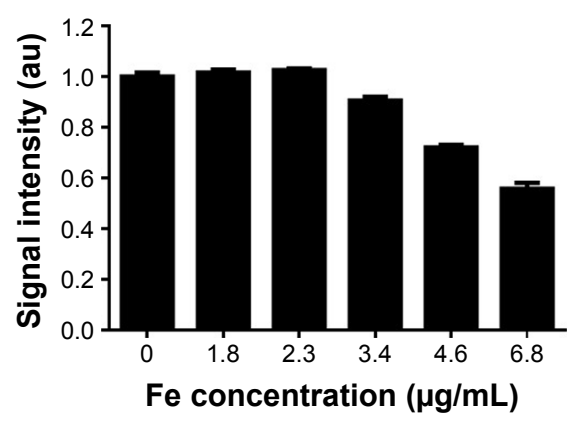

C

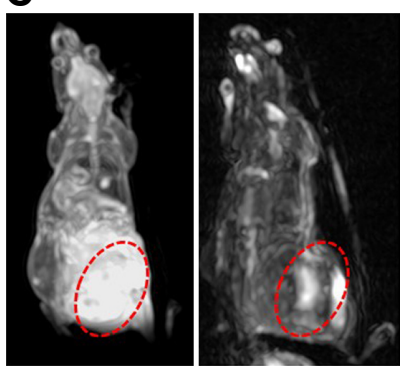

Figure $9 \mathrm{~T}_{2}$-weighted MR images and quantitative signal intensity analysis of LDM-PLGA/PPF.

Notes: (A) $T_{2}$-weighted MR images and color $T_{2}$-weighted MR images of HeLa cells treated with LDM-PLGA/PPF at nanoparticle concentrations of 80, I00, I50, 200, and $300 \mu \mathrm{g} / \mathrm{mL}$ (or Fe concentrations of I.8, 2.3, 3.4, 4.6, and $6.8 \mu \mathrm{g} / \mathrm{mL}$ ) for $12 \mathrm{~h}$. The color bar change from red to blue indicates the gradual decrease of $\mathrm{MR}$ signal intensity. (B) Quantitative signal intensity analysis. (C) In vivo $T_{2}$-weighted MR images of EMT-6 tumor-bearing mice before (left) and after (right) injection of LDM-PLGA/PPF. Tumors are marked by the red circle.

Abbreviations: MR, magnetic resonance; PLGA, poly(D,L-lactic-co-glycolic acid); PPF, PEI-PEG-FA; PEI-PEG-FA, polyethyleneimine premodified with polyethylene glycolfolic acid.

intensities of CdSe/ZnS QDs increased with the increase in L-PLGA/PPF concentration, suggesting that the imaging effects also increased. The in vivo fluorescence imaging of EMT-6 tumor-bearing nude mice as a function of time after intratumoral injection of L-PLGA/PPF hybrid nanoparticles (Figure 10) showed that, compared with the control group with injection of saline, a remarkable fluorescence signal from the nanoparticles was observed in the tumor region at $0.5 \mathrm{~h}$ after intratumoral injection. Further, this can be obviously distinguished from the surrounding normal tissues. Additionally, although there was a slight fluorescence signal intensity decrease at $2.5 \mathrm{~h}$ after injection of L-PLGA/ PPF, the fluorescence signal was still strong in the tumor

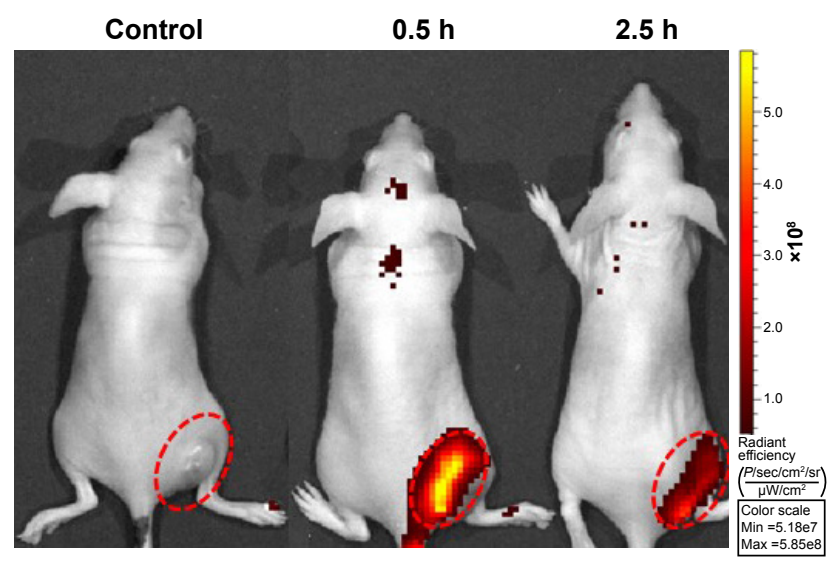

Figure 10 In vivo fluorescence imaging of EMT-6 tumor-bearing mice after intratumoral injection of saline (control) or L-PLGA/PPF nanoparticles at 0.5 and $2.5 \mathrm{~h}$. Notes: The fluorescence signal excitation from $\mathrm{CdSe} / \mathrm{ZnS}$ quantum dot incorporation into PLGA-based nanoparticles in tumor sites was strong, whereas no apparent fluorescent signal was observed in control mice. The color bar change from red to yellow indicates the gradual increase of fluorescence signal intensity. Tumors are marked by the red circle.

Abbreviations: PPF, PEI-PEG-FA; PEI-PEG-FA, polyethyleneimine premodified with polyethylene glycol-folic acid; PLGA, poly(D,L-lactic-co-glycolic acid). site and could be easily identified. These data validated the maintenance of the fluorescent PLGA-based nanoparticles in tumor tissues for at least several hours and showed the promising potential of the system as a fluorescence imaging nano-probe for theranostic applications.

\section{VEGF gene silencing efficiency in vitro}

VEGF is considered to be the most important cytokine in angiogenesis of endothelial cells. ${ }^{16}$ Here, RNAi was used against the $V E G F$ gene via targeted double-stranded RNA downregulation of $V E G F$ gene expression. HeLa cells were used to investigate the silencing efficacy of VEGF shRNA. To eliminate the interference of DOX-induced apoptosis with the silencing efficacy evaluation, singly loaded VEGF shRNA PLGA nanocomposites were used in the gene silencing assay. VEGF mRNA levels and protein levels were detected by qPCR and ELISA assays, respectively. Cells incubated with PLGA/PPF/VEGF shRNA showed a decrease in VEGF mRNA levels compared with the control and PLGA/PPF with SC shRNA (Figure 11A). Although commercial transfection reagents Lipofectamine 2000 with VEGF shRNA exhibited a higher VEGF downregulation effect, apparent cytotoxicity could be caused by Lipofectamine 2000 at relatively high dosages, consistent with previous reports. ${ }^{66}$ The expression of VEGF at the protein level was further measured by ELISA as shown in Figure 11B and was similar to the results obtained by qPCR. PLGA/PPF/VEGF shRNA showed a knockdown of VEGF expression $(3,765 \mathrm{pg} / \mathrm{mL})$ in comparison with the control $(4,236 \mathrm{pg} / \mathrm{mL})$ and PLGA/PPF/SC shRNA $(4,411 \mathrm{pg} / \mathrm{mL})$ groups. These results proved that the PLGA/PPF/VEGF shRNA nanocomposites could be utilized as an effective 
A

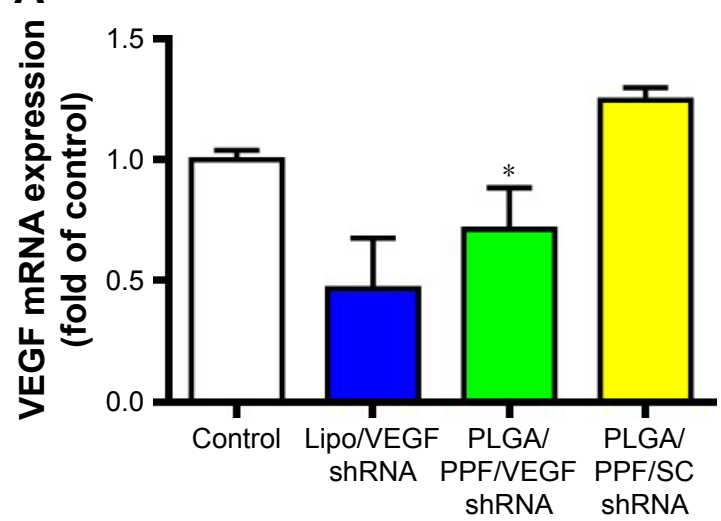

B

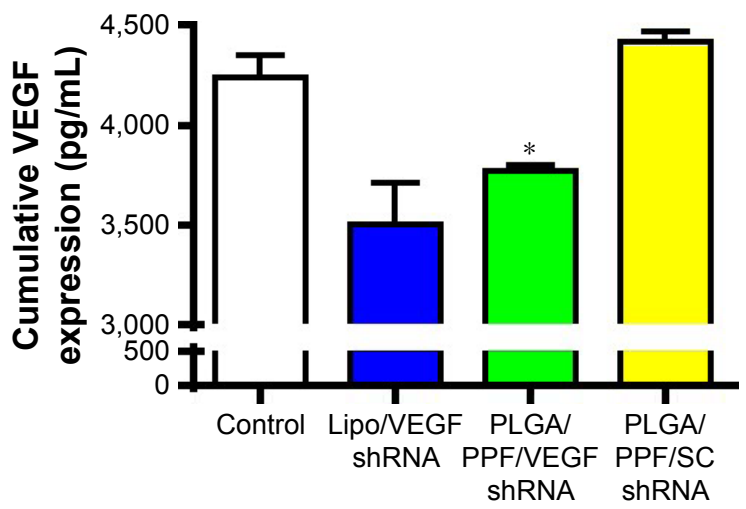

Figure II VEGF gene expression.

Notes: (A) The expression of VEGF at the mRNA level in culture media determined by quantitative real-time PCR. (B) The expression of VEGF at the protein level in culture media detected by human VEGF ELISA kit. Both experiments were conducted for a further $72 \mathrm{~h}$ after transfection with Lipo/VEGF shRNA, PLGA/PPF/VEGF shRNA, or PLGA/PPF/SC shRNA. The weight ratio of nanocomposites to SC shRNA or VEGF shRNA was I50:I. *P<0.05 compared with control.

Abbreviations: PLGA, poly(D,L-lactic-co-glycolic acid); PPF, PEI-PEG-FA; PEI-PEG-FA, polyethyleneimine premodified with polyethylene glycol-folic acid; SC shRNA, scrambled small hairpin RNA; VEGF, vascular endothelial growth factor.

gene delivery system for targeted $V E G F$ gene silencing in a highly sequence-specific fashion.

\section{Antitumor activity in vivo}

The antitumor effect/therapeutic efficacy of LDM-PLGA/ PPF/VEGF shRNA nanocomposites was then evaluated in a subcutaneous EMT-6 tumor xenograft model. Similar to saline as the control group, tumors treated with non-drugand non-shRNA-loaded PLGA nanoparticles (LM-PLGA/ PPF) grew fast, resulting in a 17.8-fold increase of tumor volumes after 21 days postinjection, which had no effect on tumor growth (Figures 12A and S6). LM-PLGA/PPF/ VEGF shRNA exhibited a slight/moderate tumor growth inhibition effect (9.6-fold increase of tumor volumes after 21 days postinjection), suggesting that interference against the $V E G F$ gene could suppress tumor growth in vivo. Additionally, LDM-PLGA/PPF/SC shRNA displayed considerable tumor growth inhibition due to the continuous release of DOX in tumor tissues and increased intratumor drug accumulation. Importantly, the most significant antitumor activity was accomplished by administration of codelivery VEGF shRNA and DOX (LDM-PLGA/ PPF/VEGF shRNA), with only 3.3-fold increase of tumor volumes after 21 days postinjection and a tumor volume decrease by $81.07 \%$ compared to the saline group. The synergistic antitumor effects of DOX and VEGF shRNA could be attributed to the induction of cell apoptosis by DOX combined with downregulation of $V E G F$ gene. Additionally, no significant body weight loss or obvious body weight fluctuations were observed among mice after the administration of different formulations during the experimental period
(Figure 12B), indicating that the there was no apparent systemic toxicity caused by the different nanocomposites. Following sacrifice of the mice at 21 days, tumors were excised, imaged, and weighed (Figure 12C and D). Consistent with the tumor growth profiles, the mean weight of the tumors in the LDM-PLGA/PPF/VEGF shRNA-treated group was the lowest among all groups and only reached $0.89 \mathrm{~g}$ compared with tumors from the control group, which reached $3.30 \mathrm{~g}$.

To further examine the therapeutic efficacy of various nanocomposites in vivo, both H\&E staining and TUNEL assays were performed to study the histopathology/ morphology and apoptosis of the tumor tissues, respectively. The results confirmed that the mice tumors treated with LDM-PLGA/PPF/VEGF shRNA resulted in more apparent coagulative necrosis as observed by morphological changes, whereas tumors in the saline- and LM-PLGA/PPF-treated groups retained a normal morphology and necrotic areas were hardly observed (Figure 12E). Furthermore, tumors on mice treated with LDM-PLGA/PPF/SC shRNA or LMPLGA/PPF/VEGF shRNA exhibited less necrotic areas. Additionally, H\&E staining was performed to examine any potential toxicity to major organs, including the heart, liver, spleen, lungs, and kidneys, on tumor-bearing mice after treatment with the various nanocomposites; nevertheless, no significant morphological changes of major organs were observed (Figure S7) nor was there any obvious histological damage in major organs, indicating that the synthesized nanocomposites have no side effects on normal tissues and low systemic toxicity at the tested dosage. More intense TUNEL signals (yellow-green fluorescence) were observed 

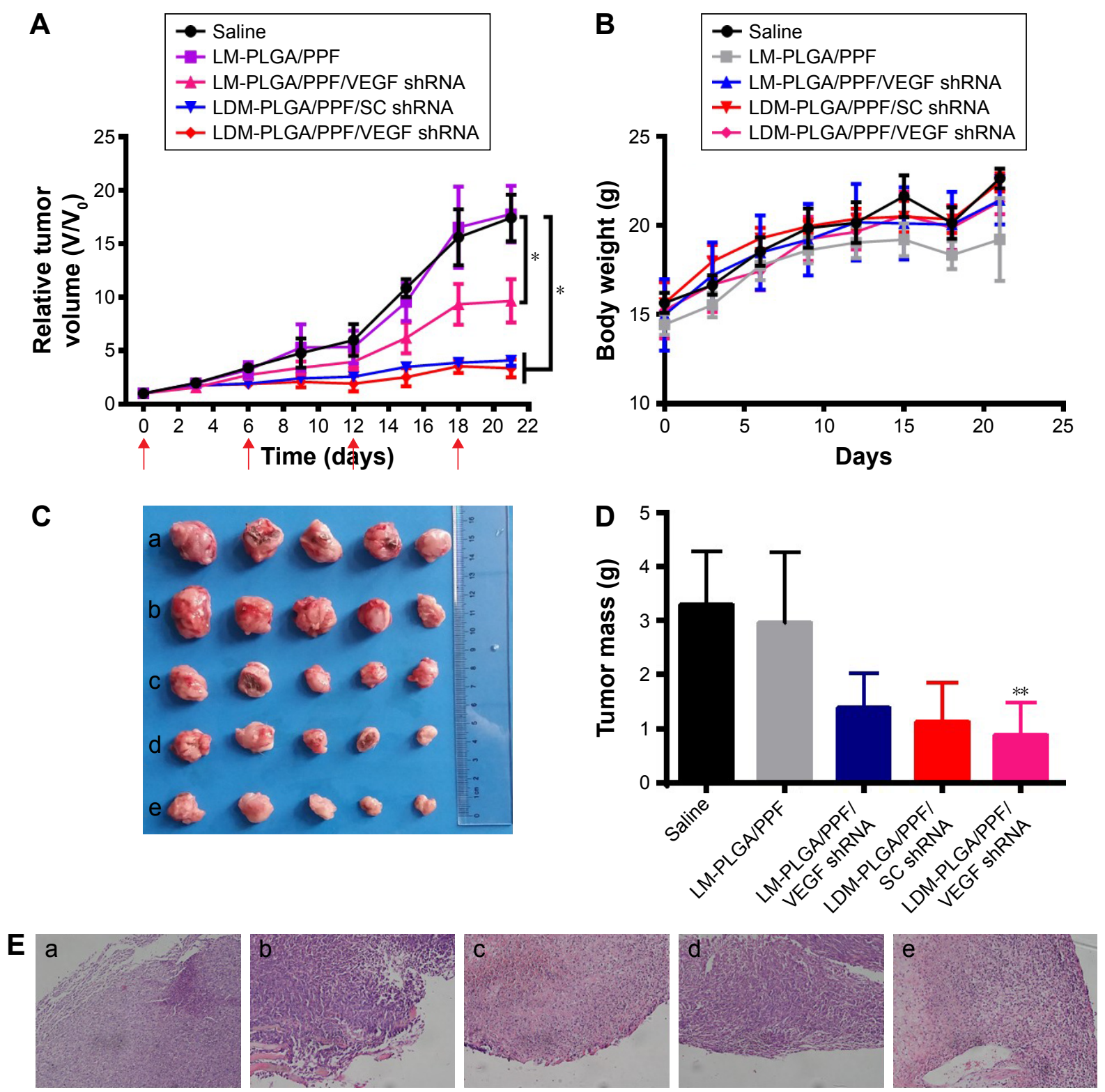

Figure 12 In vivo antitumor activity.

Notes: (A) Tumor growth curves of the various treatment groups, with the treatment schedule indicated by the red arrows. (B) Body weight changes in mice following the various treatments. (C) Optical images of tumors of different treatment groups: (a) saline, (b) LM-PLGA/PPF, (c) LM-PLGA/PPF/VEGF shRNA, (d) LDM-PLGA/PPF/SC shRNA, and (e) LDM-PLGA/PPF/VEGF shRNA. (D) Weight of dissected tumor tissues from the mice of five groups after the last treatment and sacrifice. (E) Representative H\&E-stained tumor sections from the mice of the five treatment groups: (a) saline, (b) LM-PLGA/PPF, (c) LM-PLGA/PPF/VEGF shRNA, (d) LDM-PLGA/PPF/SC shRNA, and (e) LDM-PLGA/PPF/VEGF shRNA on BALB/c mice bearing EMT-6 tumors after the last treatment and sacrifice. $* P<0.05$ and $* * P<0.01$ compared with the control (saline treatment).

Abbreviations: PLGA, poly(D,L-lactic-co-glycolic acid); PPF, PEI-PEG-FA; PEI-PEG-FA, polyethyleneimine premodified with polyethylene glycol-folic acid; shRNA, small hairpin RNA; VEGF, vascular endothelial growth factor.

in LDM-PLGA/PPF/VEGF shRNA-treated tumor tissues than in those treated with saline or the two other nanocomposites (Figure S8), substantiating that the codelivery system induced remarkable apoptosis of tumor cells, in accordance with the tumor growth curves. These results confirm that the developed co-delivery systems are capable of continuous DOX release in tumor tissues, and are therefore capable of tumor cell killing without any damage to major organs. This, in combination with the reduction of $V E G F$ gene expression, shows that the nanoparticles are potential candidates for more effective in vivo cancer therapy applications.

\section{Conclusion}

Herein, we have successfully designed and constructed a DOX and VEGF shRNA codelivery system based on magnetic and fluorescent PLGA nanoparticles and PPF 
modification. LDM-PLGA nanoparticles were fabricated using a double emulsion solvent evaporation method and coated with a PPF polymer, followed by electrostatic adsorption of VEGF shRNA. The non-drug-loaded nanoparticles (LM-PLGA/PPF) exhibited negligible hemolysis against blood cells and very low cytotoxicity against HUVECs and HeLa cells. Furthermore, the developed nanoparticles were able to enter cells via the FA receptormediated pathway and showed a continuous drug release profile and prolonged drug accumulation in an acidic microenvironment, followed by escape from the endosome/lysosome to the cytoplasm. LDM-PLGA/PPF/VEGF shRNA nanocomposites can codeliver DOX and VEGF shRNA into tumor cells and effectively kill tumor cells, suppressing $V E G F$ gene expression. Additionally, the luminescent/magnetic hybrid LDM-PLGA/PPF/VEGF shRNA nanocomposites can be used as a dual-modality imaging nano-probe for enhanced $T_{2}$-weighted MR imaging and tumor fluorescence imaging both in vitro and in vivo. The current work validates the potential of the developed multifunctional PLGA nanocomposites as an effective theranostic agent for both codelivery of drugs/genes and dual-modality imaging in cancer therapy.

\section{Acknowledgments}

We would like to thank the National Natural Science Foundation of China (31470959, 81671821, 81471785, 11272083, 31470906, and 11502049), the Sichuan Youth Science and Technology Foundation of China (2014JQ0008), the Basic Research Program of Sichuan Science and Technology Foundation (2017JY0019), the China Postdoctoral Science Foundation (2016M592657), and the Fundamental Research Funds for the Central Universities (ZYGX2015J143 and ZYGX2016001) for financial support.

\section{Disclosure}

The authors report no conflicts of interest in this work.

\section{References}

1. Siegel RL, Miller KD, Jemal A. Cancer statistics, 2015. CA Cancer J Clin. 2015;65(1):5-29.

2. Bray F, Jemal A, Grey N, Ferlay J, Forman D. Global cancer transitions according to the Human Development Index (2008-2030): a populationbased study. Lancet Oncol. 2012;13(8):790-801.

3. Ferlay J, Shin HR, Bray F, Forman D, Mathers C, Parkin DM. Estimates of worldwide burden of cancer in 2008: GLOBOCAN 2008. Int J Cancer. 2010;127(12):2893-2917.

4. Dong D, Gao W, Liu Y, Qi XR. Therapeutic potential of targeted multifunctional nanocomplex co-delivery of siRNA and low-dose doxorubicin in breast cancer. Cancer Lett. 2015;359(2):178-186.

5. Zhang P, Li J, Ghazwani M, et al. Effective co-delivery of doxorubicin and dasatinib using a PEG-Fmoc nanocarrier for combination cancer chemotherapy. Biomaterials. 2015;67:104-114.
6. Zhang Y, Li P, Pan H, et al. Retinal-conjugated $\mathrm{pH}$-sensitive micelles induce tumor senescence for boosting breast cancer chemotherapy. Biomaterials. 2016;83:219-232.

7. Jia Y, Yuan M, Yuan H, et al. Co-encapsulation of magnetic $\mathrm{Fe}_{3} \mathrm{O}_{4}$ nanoparticles and doxorubicin into biodegradable PLGA nanocarriers for intratumoral drug delivery. Int J Nanomedicine. 2012;7:1697-1708.

8. Wang YC, Wang F, Sun TM, Wang J. Redox-responsive nanoparticles from the single disulfide bond-bridged block copolymer as drug carriers for overcoming multidrug resistance in cancer cells. Bioconjug Chem. 2011;22(10):1939-1945.

9. Kajimoto K, Sato Y, Nakamura T, Yamada Y, Harashima H. Multifunctional envelope-type nano device for controlled intracellular trafficking and selective targeting in vivo. J Control Release. 2014;190:593-606.

10. Chen S, Hao X, Liang X, et al. Inorganic nanomaterials as carriers for drug delivery. J Biomed Nanotechnol. 2016;12(1):1-27.

11. Li J, Zheng L, Cai H, et al. Polyethyleneimine-mediated synthesis of folic acid-targeted iron oxide nanoparticles for in vivo tumor MR imaging. Biomaterials. 2013;34(33):8382-8392.

12. Cai H, An X, Cui J, et al. Facile hydrothermal synthesis and surface functionalization of polyethyleneimine-coated iron oxide nanoparticles for biomedical applications. ACS Appl Mater Interfaces. 2013; 5(5):1722-1731.

13. Wen S, Zhao Q, An X, et al. Multifunctional PEGylated multiwalled carbon nanotubes for enhanced blood pool and tumor MR imaging. Adv Healthc Mater. 2014;3(10):1568-1577, 1525.

14. Swanson SD, Kukowska-Latallo JF, Patri AK, et al. Targeted gadoliniumloaded dendrimer nanoparticles for tumor-specific magnetic resonance contrast enhancement. Int J Nanomedicine. 2008;3(2):201-210.

15. Li J, Shi X, Shen M. Hydrothermal synthesis and functionalization of iron oxide nanoparticles for MR imaging applications. Part Part Syst Char. 2014;31(12):1223-1237.

16. Wang SH, Shi X, Van Antwerp M, et al. Dendrimer-functionalized iron oxide nanoparticles for specific targeting and imaging of cancer cells. Adv Funct Mater. 2007;17(16):3043-3050.

17. Neuberger T, Schöpf B, Hofmann H, Hofmann M, von Rechenberg B. Superparamagnetic nanoparticles for biomedical applications: Possibilities and limitations of a new drug delivery system. J Magn Magn Mater. 2005;293(1):483-496.

18. Song X, Luo X, Zhang Q, Zhu A, Ji L, Yan C. Preparation and characterization of biofunctionalized chitosan $/ \mathrm{Fe}_{3} \mathrm{O}_{4}$ magnetic nanoparticles for application in liver magnetic resonance imaging. JMagn Magn Mater. 2015;388:116-122.

19. Wang J, Chen Y, Chen B, et al. Pharmacokinetic parameters and tissue distribution of magnetic $\mathrm{Fe}_{3} \mathrm{O}_{4}$ nanoparticles in mice. Int $J$ Nanomedicine. 2010;5:861-866.

20. Lin Y, Zhang L, Yao W, et al. Water-soluble chitosan-quantum dot hybrid nanospheres toward bioimaging and biolabeling. ACS Appl Mater Interfaces. 2011;3(4):995-1002.

21. Medintz IL, Uyeda HT, Goldman ER, Mattoussi H. Quantum dot bioconjugates for imaging, labelling and sensing. Nat Mater. 2005;4(6): 435-446.

22. Gao ZF, Li TT, Xu XL, et al. Green light-emitting polyepinephrinebased fluorescent organic dots and its application in intracellular metal ions sensing. Biosens Bioelectron. 2016;83:134-141.

23. Cho HS, Dong Z, Pauletti GM, et al. Fluorescent, superparamagnetic nanospheres for drug storage, targeting, and imaging: a multifunctional nanocarrier system for cancer diagnosis and treatment. ACS Nano. 2010; 4(9):5398-5404.

24. Shen JM, Xu L, Lu Y, et al. Chitosan-based luminescent/magnetic hybrid nanogels for insulin delivery, cell imaging, and antidiabetic research of dietary supplements. Int J Pharm. 2012;427(2):400-409.

25. Zhang H, Yee D, Wang C. Quantum dots for cancer diagnosis and therapy: biological and clinical perspectives. Nanomedicine (Lond). 2008; 3(1):83-91.

26. Huang X, Jain PK, El-Sayed IH, El-Sayed MA. Gold nanoparticles: interesting optical properties and recent applications in cancer diagnostics and therapy. Nanomedicine (Lond). 2007;2(5):681-693. 
27. Wang H, Zhang S, Liao Z, et al. PEGlated magnetic polymeric liposome anchored with TAT for delivery of drugs across the blood-spinal cord barrier. Biomaterials. 2010;31(25):6589-6596.

28. Kim JW, Shashkov EV, Galanzha EI, Kotagiri N, Zharov VP. Photothermal antimicrobial nanotherapy and nanodiagnostics with selfassembling carbon nanotube clusters. Lasers Surg Med. 2007;39(7): 622-634.

29. Nicolas J, Mura S, Brambilla D, Mackiewicz N, Couvreur P. Design, functionalization strategies and biomedical applications of targeted biodegradable/biocompatible polymer-based nanocarriers for drug delivery. Chem Soc Rev. 2013;42(3):1147-1235.

30. Arias JL, Clares B, Morales ME, Gallardo V, Ruiz MA. Lipid-based drug delivery systems for cancer treatment. Curr Drug Targets. 2011;12(8): 1151-1165.

31. Vallet-Regi M, Balas F, Arcos D. Mesoporous materials for drug delivery. Angew Chem Int Ed Engl. 2007;46(40):7548-7558.

32. Huang S, Fu X. Naturally derived materials-based cell and drug delivery systems in skin regeneration. J Control Release. 2010;142(2) 149-159.

33. Yang H, Xu M, Li S, et al. Chitosan hybrid nanoparticles as a theranostic platform for targeted doxorubicin/VEGF shRNA codelivery and dual-modality fluorescence imaging. RSC Adv. 2016;6: 29685-29696.

34. Wang H, Wu Y, Zhao R, Nie G. Engineering the assemblies of biomaterial nanocarriers for delivery of multiple theranostic agents with enhanced antitumor efficacy. Adv Mater. 2013;25(11):1616-1622.

35. Li Z, Barnes JC, Bosoy A, Stoddart JF, Zink JI. Mesoporous silica nanoparticles in biomedical applications. Chem Soc Rev. 2012;41(7): 2590-2605.

36. Li CX, Parker A, Menocal E, Xiang S, Borodyansky L, Fruehauf JH. Delivery of RNA interference. Cell Cycle. 2006;5(18):2103-2109.

37. Ferrara N. Vascular endothelial growth factor. Arterioscler Thromb Vasc Biol. 2009;29(6):789-791.

38. Kalishwaralal K, Banumathi E, Ram Kumar Pandian S, et al. Silver nanoparticles inhibit VEGF induced cell proliferation and migration in bovine retinal endothelial cells. Colloids Surf B Biointerfaces. 2009; 73(1):51-57.

39. Folkman J. Role of angiogenesis in tumor growth and metastasis. Semin Oncol. 2002;29(6 Suppl 16):15-18.

40. Hicklin DJ, Ellis LM. Role of the vascular endothelial growth factor pathway in tumor growth and angiogenesis. J Clin Oncol. 2005; 23(5):1011-1027.

41. Claffey KP, Robinson GS. Regulation of VEGF/VPF expression in tumor cells: consequences for tumor growth and metastasis. Cancer Metastasis Rev. 1996;15(2):165-176.

42. Salva E, Kabasakal L, Eren F, Cakalagaoglu F, Ozkan N, Akbuga J. Chitosan/short hairpin RNA complexes for vascular endothelial growth factor suppression invasive breast carcinoma. Oligonucleotides. 2010; 20(4):183-190.

43. Li T, Shen X, Geng Y, et al. Folate-functionalized magnetic-mesoporous silica nanoparticles for drug/gene codelivery to potentiate the antitumor efficacy. ACS Appl Mater Interfaces. 2016;8(22):13748-13758.

44. Li T, Shen X, Chen Y, et al. Polyetherimide-grafted $\mathrm{Fe}_{3} \mathrm{O}_{4} @ \mathrm{SiO}_{2}$ nanoparticles as theranostic agents for simultaneous VEGF siRNA delivery and magnetic resonance cell imaging. Int $J$ Nanomedicine. 2015;10:4279-4291.

45. Huang HY, Kuo WT, Chou MJ, Huang YY. Co-delivery of anti-vascular endothelial growth factor siRNA and doxorubicin by multifunctional polymeric micelle for tumor growth suppression. J Biomed Mater Res A. 2011;97(3):330-338.

46. Feng Q, Yu MZ, Wang JC, et al. Synergistic inhibition of breast cancer by co-delivery of VEGF siRNA and paclitaxel via vapreotide-modified core-shell nanoparticles. Biomaterials. 2014;35(18):5028-5038.

47. El-Gogary RI, Rubio N, Wang JT, et al. Polyethylene glycol conjugated polymeric nanocapsules for targeted delivery of quercetin to folateexpressing cancer cells in vitro and in vivo. ACS Nano. 2014;8(2): 1384-1401.
48. Graf N, Bielenberg DR, Kolishetti N, et al. Alpha(v)beta(3) integrintargeted PLGA-PEG nanoparticles for enhanced anti-tumor efficacy of a Pt(IV) prodrug. ACS Nano. 2012;6(5):4530-4539.

49. Deng L, Li L, Yang H, et al. Development and optimization of doxorubicin loaded poly(lactic-co-glycolic acid) nanobubbles for drug delivery into HeLa cells. J Nanosci Nanotechnol. 2014;14(4):2947-2954.

50. Yang H, Deng L, Li T, et al. Multifunctional PLGA nanobubbles as theranostic agents: combining doxorubicin and P-gp siRNA codelivery into human breast cancer cells and ultrasound cellular imaging. J Biomed Nanotechnol. 2015;11(12):2124-2136.

51. Yang H, Li Y, Li T, et al. Multifunctional core/shell nanoparticles cross-linked polyetherimide-folic acid as efficient Notch-1 siRNA carrier for targeted killing of breast cancer. Sci Rep. 2014;4:7072.

52. Zhao X, Li F, Li Y, et al. Co-delivery of HIF1alpha siRNA and gemcitabine via biocompatible lipid-polymer hybrid nanoparticles for effective treatment of pancreatic cancer. Biomaterials. 2015;46:13-25.

53. Zhu A, Miao K, Deng Y, et al. Dually $\mathrm{pH} /$ reduction-responsive vesicles for ultrahigh-contrast fluorescence imaging and thermo-chemotherapysynergized tumor ablation. ACS Nano. 2015;9(8):7874-7885.

54. Yu J, Ju Y, Zhao L, et al. Multistimuli-regulated photochemothermal cancer therapy remotely controlled via $\mathrm{Fe}_{5} \mathrm{C}_{2}$ nanoparticles. ACS Nano. 2016;10(1):159-169.

55. Li J, He Y, Sun W, et al. Hyaluronic acid-modified hydrothermally synthesized iron oxide nanoparticles for targeted tumor MR imaging. Biomaterials. 2014;35(1):3666-3677.

56. Hao X, Hu X, Zhang C, et al. Hybrid mesoporous silica-based drug carrier nanostructures with improved degradability by hydroxyapatite. ACS Nano. 2015;9(10):9614-9625.

57. Tang S, Yin Q, Zhang Z, et al. Co-delivery of doxorubicin and RNA using $\mathrm{pH}$-sensitive poly (beta-amino ester) nanoparticles for reversal of multidrug resistance of breast cancer. Biomaterials. 2014;35(23): 6047-6059.

58. Shen M, Cai H, Wang X, et al. Facile one-pot preparation, surface functionalization, and toxicity assay of APTS-coated iron oxide nanoparticles. Nanotechnology. 2012;23(10):105601.

59. Dong DW, Xiang B, Gao W, Yang ZZ, Li JQ, Qi XR. pH-responsive complexes using prefunctionalized polymers for synchronous delivery of doxorubicin and siRNA to cancer cells. Biomaterials. 2013;34(20):4849-4859.

60. Feng W, Nie W, He C, et al. Effect of $\mathrm{pH}$-responsive alginate/chitosan multilayers coating on delivery efficiency, cellular uptake and biodistribution of mesoporous silica nanoparticles based nanocarriers. ACS Appl Mater Interfaces. 2014;6(11):8447-8460.

61. Sun C, Sze R, Zhang M. Folic acid-PEG conjugated superparamagnetic nanoparticles for targeted cellular uptake and detection by MRI J Biomed Mater Res A. 2006;78(3):550-557.

62. Kumar M, Singh G, Arora V, et al. Cellular interaction of folic acid conjugated superparamagnetic iron oxide nanoparticles and its use as contrast agent for targeted magnetic imaging of tumor cells. Int $J$ Nanomedicine. 2012;7:3503-3516.

63. Chen J, Qiu X, Ouyang J, Kong J, Zhong W, Xing MM. pH and reduction dual-sensitive copolymeric micelles for intracellular doxorubicin delivery. Biomacromolecules. 2011;12(10):3601-3611.

64. Ng CT, Tang FM, Li JJ, Ong C, Yung LL, Bay BH. Clathrin-mediated endocytosis of gold nanoparticles in vitro. Anat Rec (Hoboken). 2015; 298(2):418-427.

65. Kamba SA, Ismail M, Hussein-Al-Ali SH, Ibrahim TA, Zakaria ZA. In vitro delivery and controlled release of Doxorubicin for targeting osteosarcoma bone cancer. Molecules. 2013;18(9):10580-10598.

66. Khatri N, Baradia D, Vhora I, Rathi M, Misra A. Development and characterization of siRNA lipoplexes: effect of different lipids, in vitro evaluation in cancerous cell lines and in vivo toxicity study. AAPS PharmSciTech. 2014;15(6):1630-1643. 


\section{Supplementary materials}
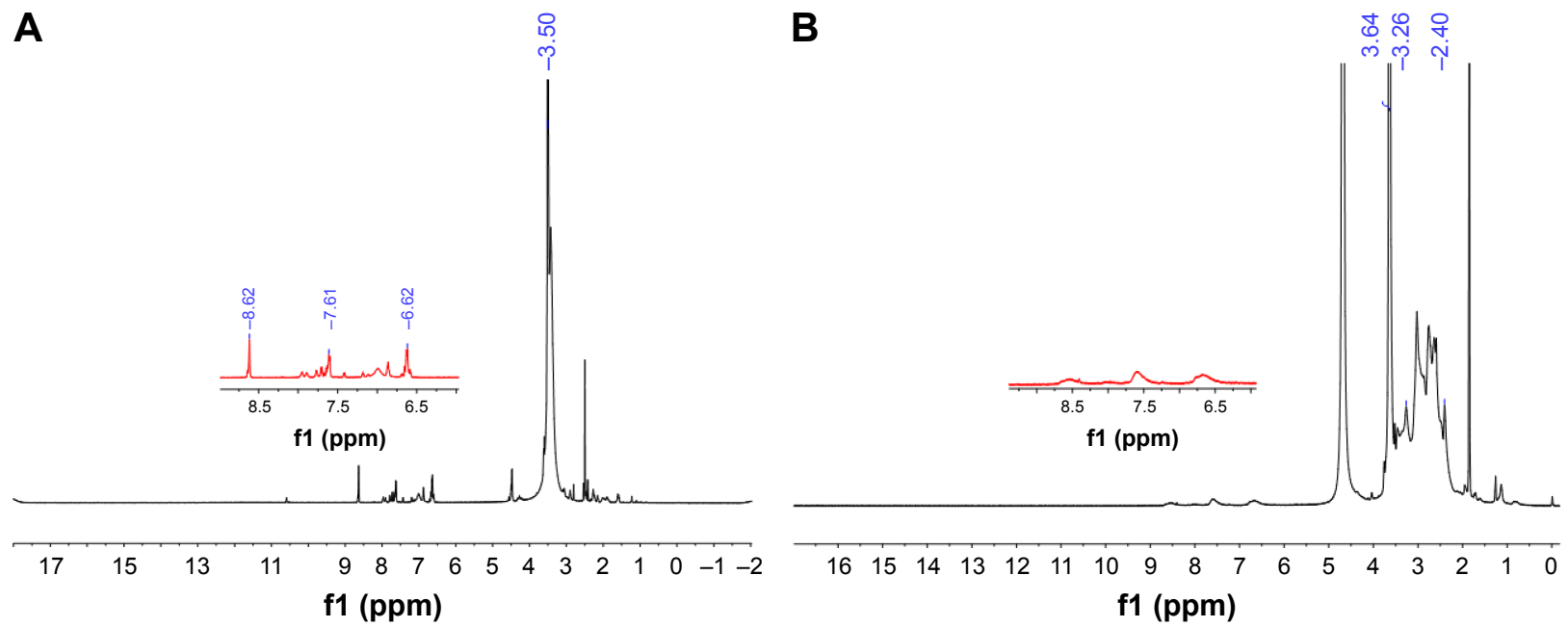

Figure SI 'H NMR spectra of PEG-FA (A) and PEI-PEG-FA (B).

Abbreviation: PEI-PEG-FA, polyethyleneimine premodified with polyethylene glycol-folic acid.
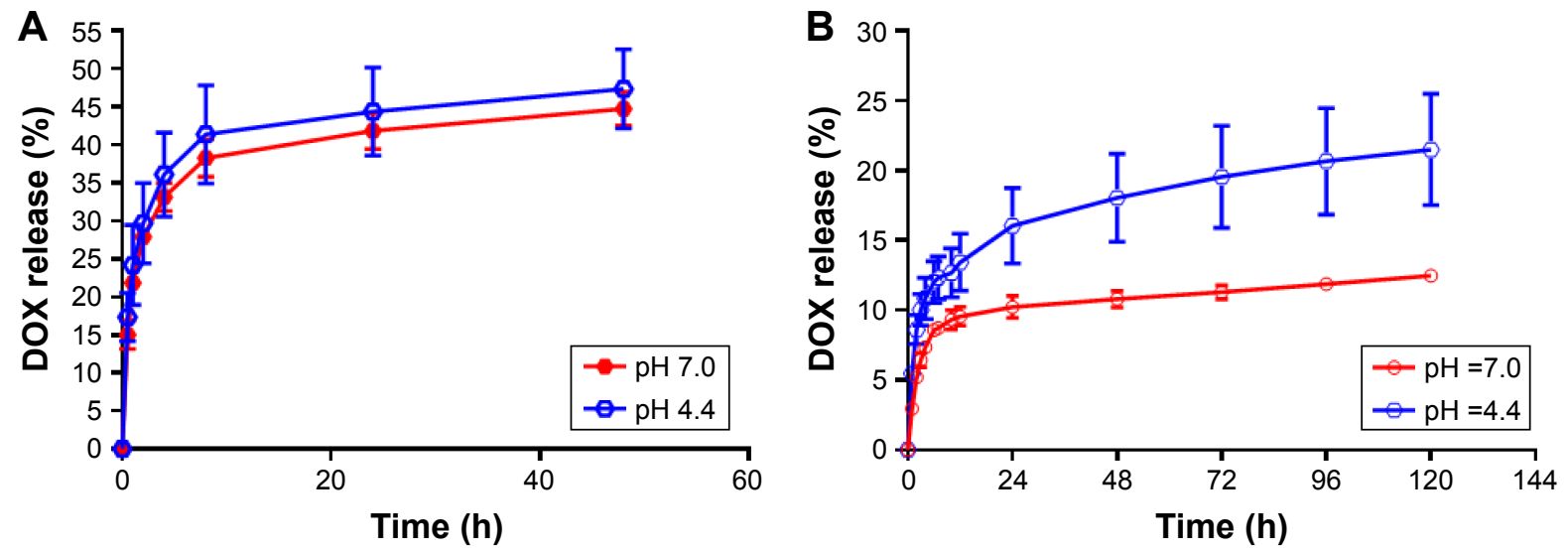

Figure S2 In vitro release of DOX from LDM-PLGA (A) and LDM-PLGA/PPF (B) under different pH conditions and time points. Drug concentration in the supernatant was assessed by measuring the absorbance at $480 \mathrm{~nm}$.

Abbreviations: DOX, doxorubicin; PLGA, poly(D,L-lactic-co-glycolic acid); PPF, PEI-PEG-FA; PEI-PEG-FA, polyethyleneimine premodified with polyethylene glycol-folic acid. 


\section{A}

\section{LDM-PLGA/PPF}

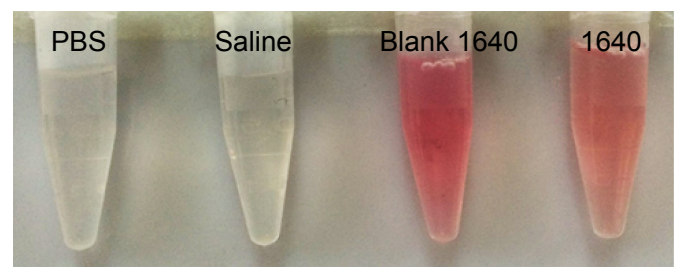

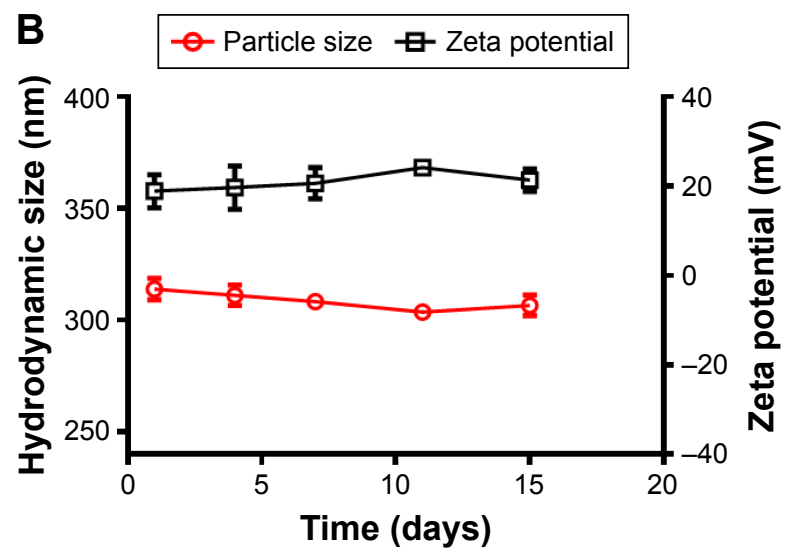

Figure S3 Stability tests.

Notes: (A) Images of LDM-PLGA/PPF dispersed in PBS, saline, serum-free RPMI I640 cell culture medium, and complete RPMI I640 cell culture medium. (B) Hydrodynamic size and zeta potential of LDM-PLGA/PPF at different time points.

Abbreviations: PLGA, poly(D,L-lactic-co-glycolic acid); PPF, PEI-PEG-FA; PEI-PEG-FA, polyethyleneimine premodified with polyethylene glycol-folic acid; PBS, phosphatebuffered solution; RPMI, Roswell Park Memorial Institute.
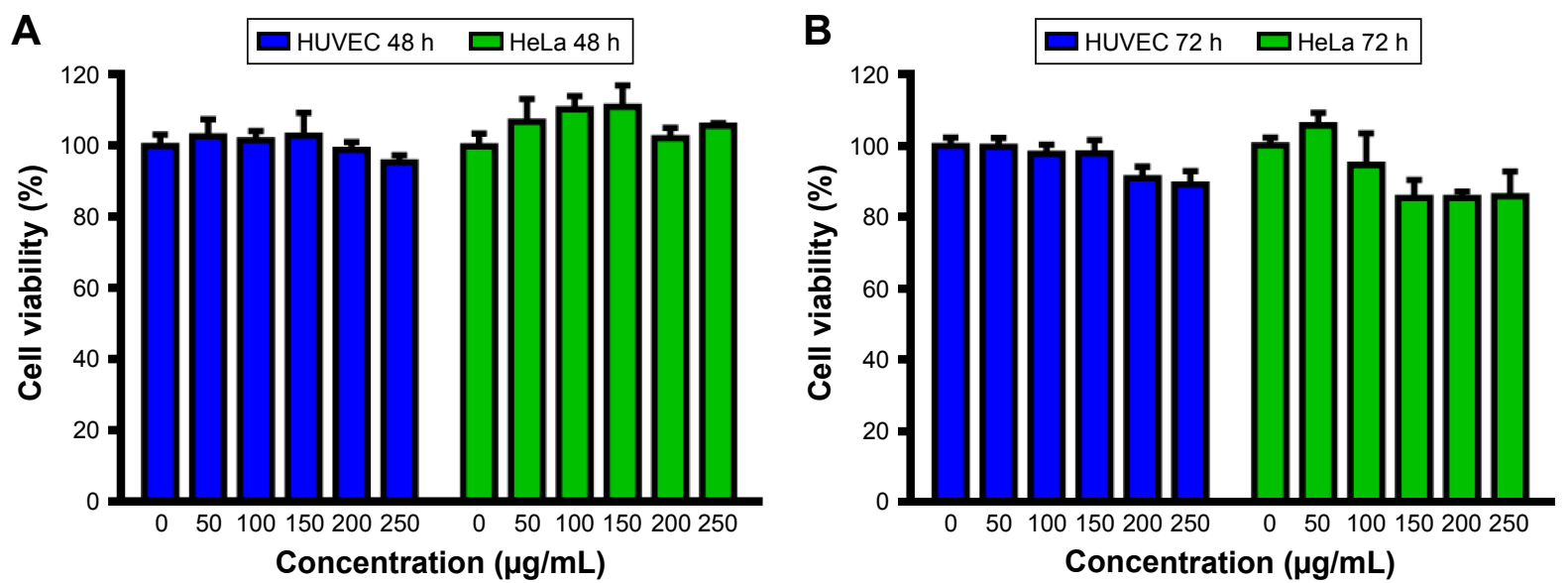

Figure S4 In vitro cell viability of HeLa cells and HUVEC after treatment LM-PLGA/PPF at the concentration of 0-250 $\mu g / \mathrm{mL}$ for 48 (A) and $72 \mathrm{~h}$ (B). Abbreviations: HUVEC, human umbilical vein endothelial cell; PLGA, poly(D,L-lactic-co-glycolic acid); PPF, PEI-PEG-FA; PEI-PEG-FA, polyethyleneimine premodified with polyethylene glycol-folic acid.

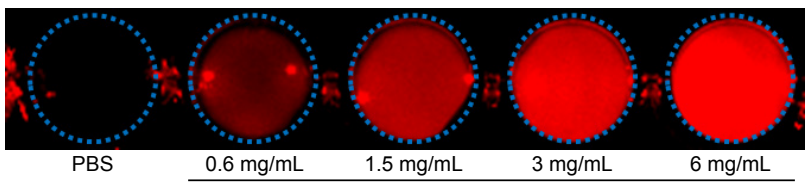

Particle con $(\mathrm{mg} / \mathrm{mL})$ L-PLGA/PPF

Figure $\mathbf{S 5}$ In vitro representative fluorescence images by activation of CdSe/ZnS quantum dots.

Abbreviations: PLGA, poly(D,L-lactic-co-glycolic acid); PPF, PEI-PEG-FA; PEI-PEG-FA, polyethyleneimine premodified with polyethylene glycol-folic acid; PBS, phosphatebuffered solution. 

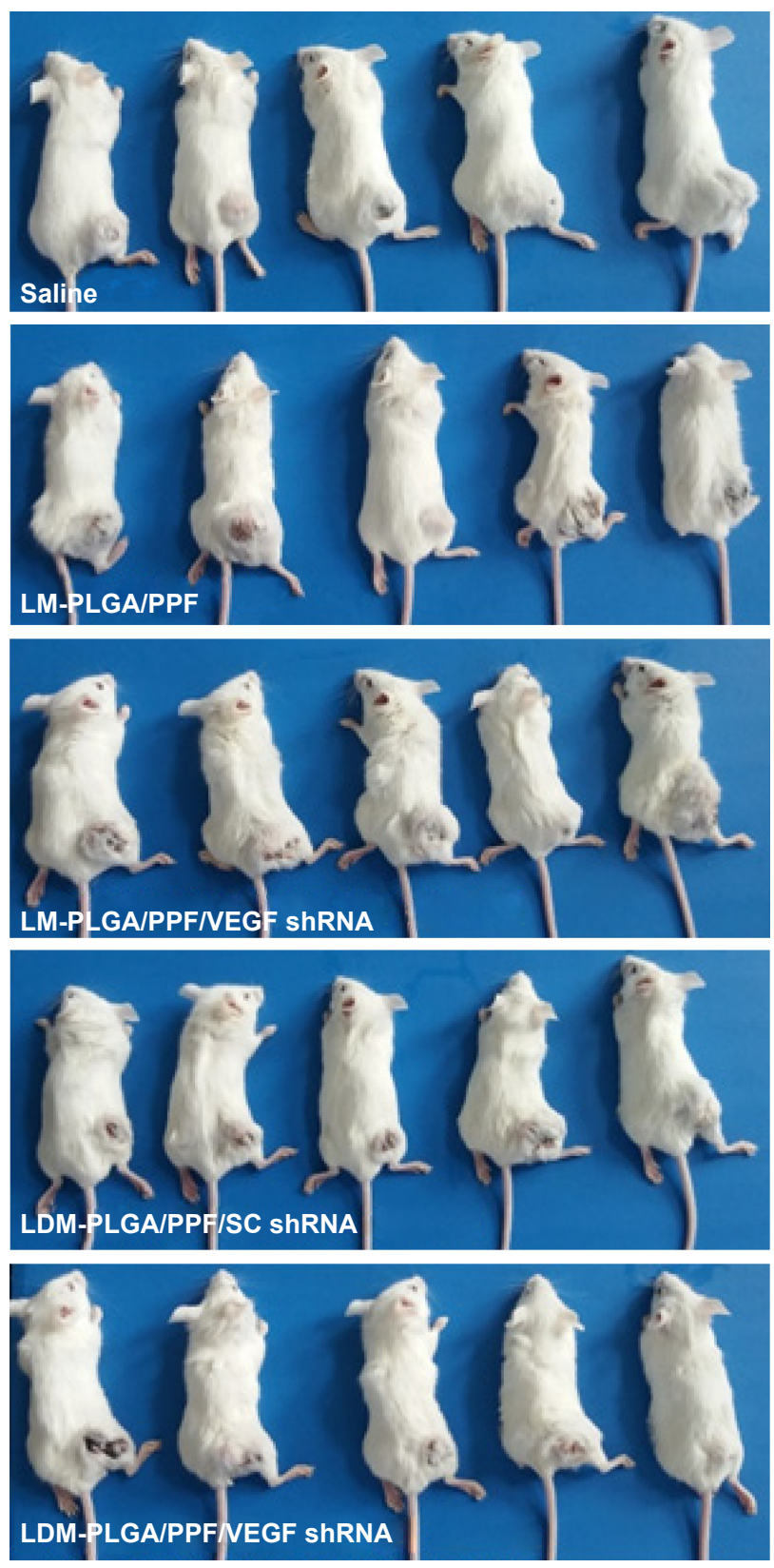

Figure S6 Photographs of different groups of tumor-bearing mice after 21-day treatment with saline, LM-PLGA/PPF, LM-PLGA/PPF/VEGF shRNA, LDM-PLGA/PPF/SC shRNA, and LDM-PLGA/PPF/VEGF shRNA.

Abbreviations: PLGA, poly(D,L-lactic-co-glycolic acid); PPF, PEI-PEG-FA; PEI-PEG-FA, polyethyleneimine premodified with polyethylene glycol-folic acid; shRNA, small hairpin RNA; VEGF, vascular endothelial growth factor. 


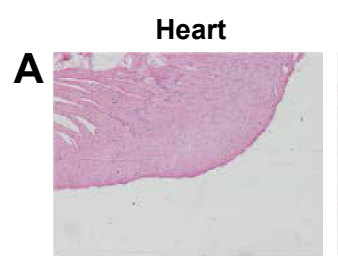

B

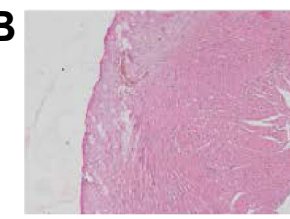

C

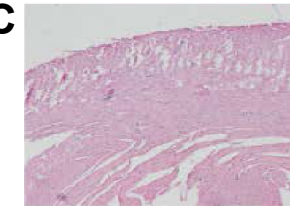

D

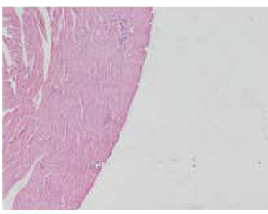

E

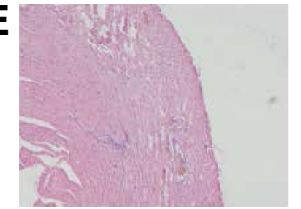

Liver
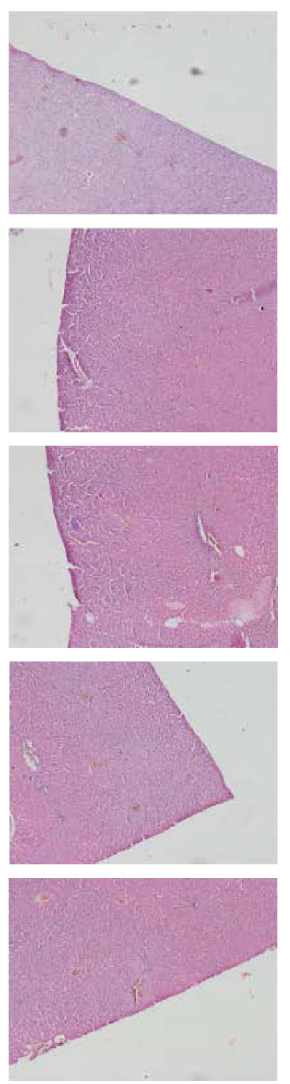

Spleen
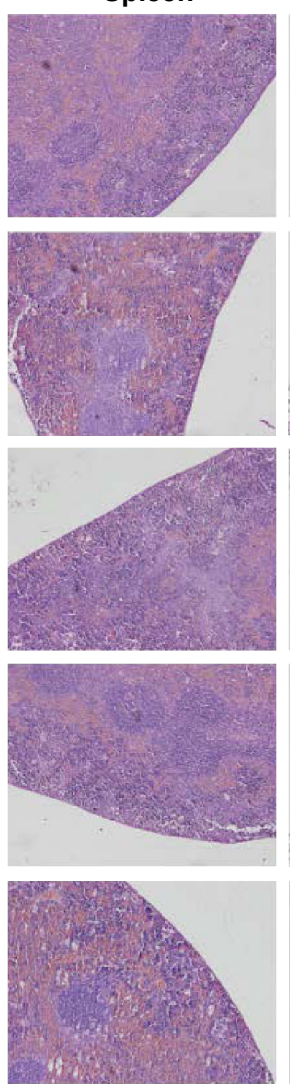
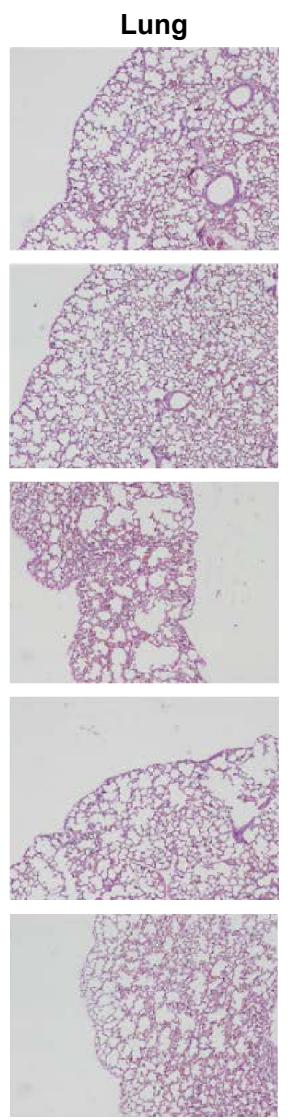

Kidney
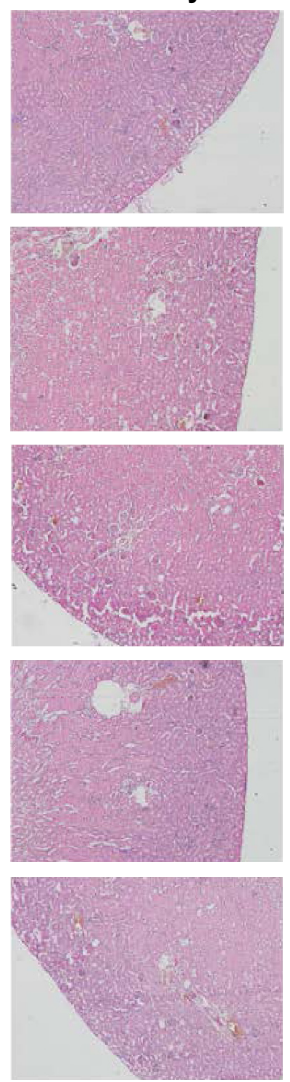

Figure S7 Representative images of H\&E-stained sections of the heart, liver, spleen, lung, and kidney collected from tumor-bearing BALB/c mice after treatment with saline (A), LM-PLGA/PPF (B), LM-PLGA/PPF/VEGF shRNA (C), LDM-PLGA/PPF/SC shRNA (D), and LDM-PLGA/PPF/VEGF shRNA (E).

Abbreviations: PLGA, poly(D,L-lactic-co-glycolic acid); PPF, PEI-PEG-FA; PEI-PEG-FA, polyethyleneimine premodified with polyethylene glycol-folic acid; SC shRNA, scrambled small hairpin RNA; VEGF, vascular endothelial growth factor.
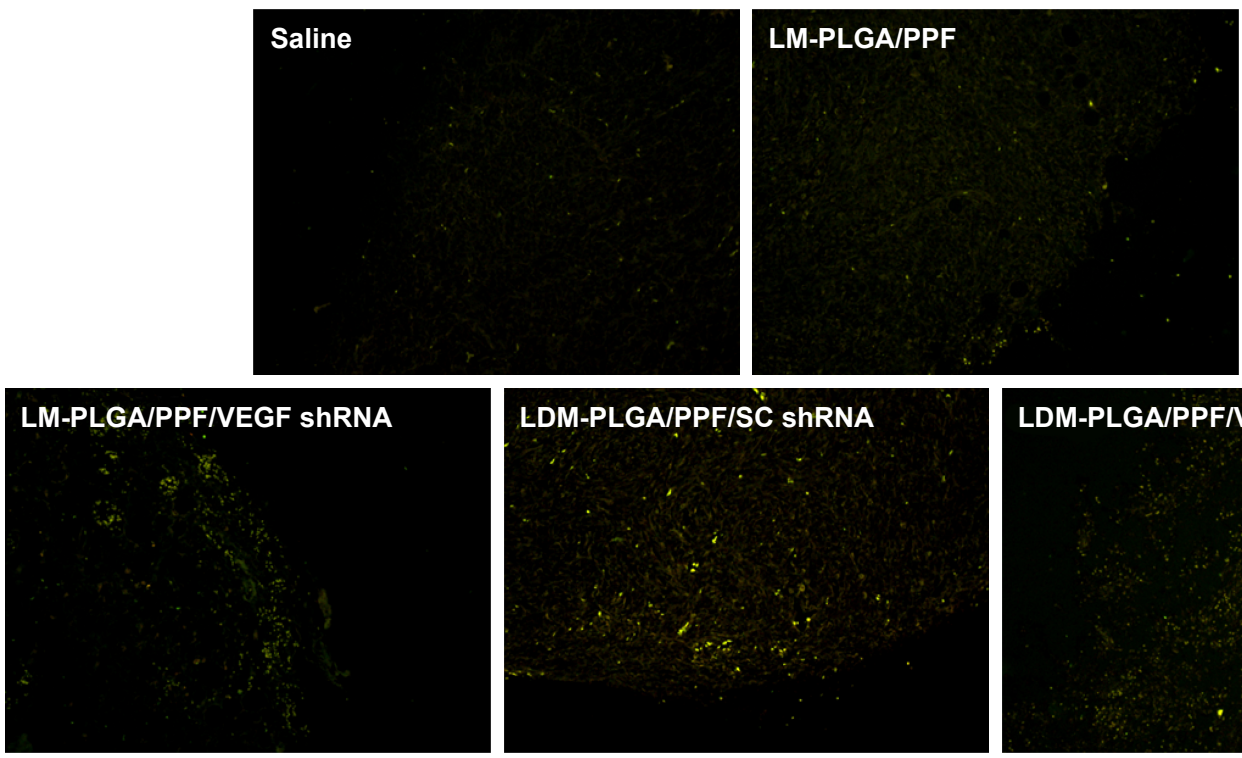
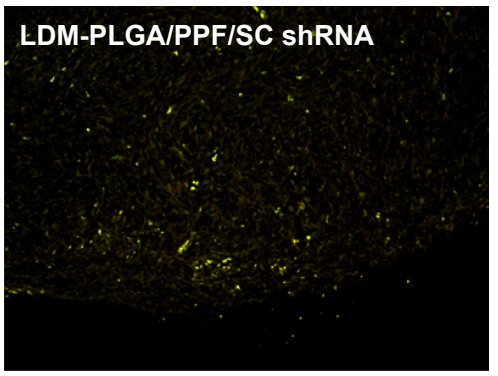

LDM-PLGA/PPF/VEGF ShRNA

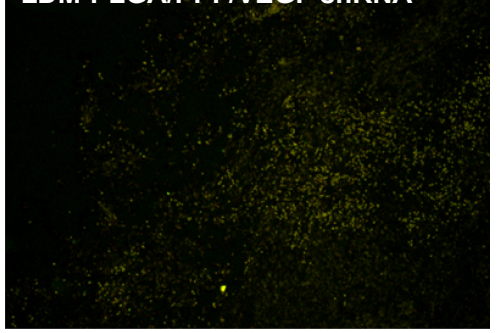

Figure $\mathbf{S} 8$ TUNEL staining of the tumors with different treatments.

Note: TUNEL-positive cells (apoptotic cells) exhibited yellow-green fluorescence.

Abbreviations: PLGA, poly(D,L-lactic-co-glycolic acid); PPF, PEI-PEG-FA; PEI-PEG-FA, polyethyleneimine premodified with polyethylene glycol-folic acid; SC shRNA, scrambled small hairpin RNA; TUNEL, terminal deoxynucleotidyl transferase dUTP nick-end labeling; VEGF, vascular endothelial growth factor. 


\section{Publish your work in this journal}

The International Journal of Nanomedicine is an international, peerreviewed journal focusing on the application of nanotechnology in diagnostics, therapeutics, and drug delivery systems throughout the biomedical field. This journal is indexed on PubMed Central, MedLine, CAS, SciSearch $\AA$, Current Contents $\AA /$ Clinical Medicine,

Journal Citation Reports/Science Edition, EMBase, Scopus and the Elsevier Bibliographic databases. The manuscript management system is completely online and includes a very quick and fair peer-review system, which is all easy to use. Visit http://www.dovepress.com/ testimonials.php to read real quotes from published authors.

Submit your manuscript here: http://www.dovepress.com/international-journal-of-nanomedicine-journal 